\title{
Linearly polarized gluons in charmonium and bottomonium production in color octet model
}

\author{
Asmita Mukherjee and Sangem Rajesh \\ Department of Physics, Indian Institute of Technology Bombay, Mumbai-400076, India.
}

(Dated: July 15, 2021)

\begin{abstract}
We study the possibility to probe the unpolarized and linearly polarized transverse momentumdependent gluon distributions in unpolarized $p p$ collision in charmonium and bottomonium production, employing non-relativistic QCD (NRQCD) based color octet model within transverse momentum dependent (TMD) factorization framework. The transverse momentum $\left(p_{T}\right)$ and rapidity distributions of $J / \psi$ and $\Upsilon(1 \mathrm{~S})$ at LHCb, RHIC and AFTER energies are estimated. Significant modulations in transverse momentum spectrum of quarkonium in the low $p_{T}$ region is obtained when contributions of linearly polarized gluons inside an unpolarized proton are taken into account. The results of quarkonium production in color octet model and color evaporation model are compared.
\end{abstract}




\section{INTRODUCTION}

Transverse momentum dependent (TMD) parton distribution functions (PDFs) and fragmentation functions (FFs), in short TMDs play an essential role in understanding the spin and spatial structure of the proton. TMDs depend on both longitudinal momentum fraction $x$ and internal transverse momentum $k_{\perp}$ carried by partons in contrast to traditional collinear parton distributions (PDFs). TMDs being nonperturbative objects have to be extracted from experimental data. The data mainly comes from two types of experiments, semi-inclusive deep inelastic scattering (SIDIS) and Drell-Yan (DY) process. In these experiments one measures the transverse momentum of final observed hadron which is sensitive to the intrinsic transverse momentum $\left(k_{\perp}\right)$ of the parton.

TMD factorization (generalized factorization) allows us to relate the cross section in terms of convolution of TMDs and hard scattering factor, which can be calculated in order of $\alpha_{s}$. TMD factorization has been studied extensively for SIDIS, DY and $e^{+} e^{-}$annihilation process [1] at

small transverse momentum $\left(p_{T}\right)$ of the observed hadron i.e., $\Lambda_{Q C D} \ll p_{T} \ll Q$, where $Q$ is the hard scale of the process. There are interesting issues related to the process dependence of the TMDs and the applicability of factorization for more general processes. Also an alternative approach to understand the single spin asymmetries is based on collinear twist-3 PDFs [2, 3]. At small transverse momentum, the radiative gluon emissions become important which need to be resummed up to all orders in $\alpha_{s}$. This is accomplished through TMD evolution equation. Thus the TMDs satisfy a different and more involved evolution equation compared to the standard collinear PDFs. The evolution kernel is calculated using perturbation theory which is independent of the process chosen and type of TMDs. TMD evolution equation, which can be obtained by solving renormalization group ( $R G)$ and Collins-Soper [1] equations, is the consequence of TMD factorization. There is a non-perturbative part of the evolution kernel which is usually parametrized.

Within generalized factorization framework, the low $p_{T}$ region $\left(p_{T} \approx \mathcal{O}(10 \mathrm{GeV})\right)$ in differential cross section of $Z$-boson in $p p$ collision at $\sqrt{s}=8 \mathrm{TeV}$ (LHC) [4] and $\sqrt{s}=1.8 \mathrm{TeV}(\mathrm{CDF})$ [5] could be predicted to a good accuracy. The reason is the resummation of large logarithmic corrections. However, the usual collinear factorization applied with convoluted collinear PDFs 
fails to describe the low $p_{T}$ region of $Z$-boson spectrum. In this formalism, radiative corrections make the cross section diverge as $p_{T}$ decreases [4]. In recent past, much work has been done to extract the quark TMDs from experiments at COMPASS, HERMES and JLab [6, 7]. However, very limited information is available about gluon TMDs experimentally. To understand TMDs fully, we need global analysis of SIDIS and DY data. Nevertheless, the difficulty arises since the SIDIS and DY data span over different ranges in $p_{T}$ and $\sqrt{s}$ [8]. In Higgs boson production at NNLO [9], the polarized gluons contribute dominantly over the unpolarized gluons because of the fact that polarized gluons are generated from unpolarized gluons.

In Ref. [10], the authors pointed out that linearly polarized gluons can exist even at tree level inside an unpolarized hadron. The associated density function denoted by $h_{1}^{\perp g}$, represents the probability of finding the linearly polarized gluon inside an unpolarized hadron. For the existence of $h_{1}^{\perp g}$, gluons should have non-zero transverse momentum with respect to hadron. $h_{1}^{\perp g}$ being a time-reversal even (T-even) TMD, initial/final state interactions are not necessary for the presence of $h_{1}^{\perp g}$. The gluon-gluon correlator [10] of spin- $\frac{1}{2}$ unpolarized proton is parametrized in terms of unpolarized density of gluons, $f_{1}^{g}$ and $h_{1}^{\perp g}$. These are the only two TMDs of unpolarized proton which describe the transverse dynamics of gluons. Without prior understanding of these functions, it is not feasible to have theoretical interpretation of the physical quantities that are obtained experimentally. However, the studies on these functions are still sparse. Hence, the determination of these functions must be of prime importance.

Though, the experimental investigations on the quantification of $h_{1}^{\perp g}$ are not carried out so far, a model independent theoretical upper bound is given in Ref. [11]. However, a lot of theoretical works have suggested to probe $h_{1}^{\perp g}$ in several ways. Heavy quark pair or dijet production in SIDIS [12], diphoton pair [13] and $\Upsilon(1 \mathrm{~S})+$ jet [14] production in $p p$ collision have been suggested to probe $h_{1}^{\perp g}$. In these processes it has been shown that $h_{1}^{\perp g}$ can be probed by measuring azimuthal asymmetries. Moreover, the participation of two linearly polarized gluons in the scattering process results in a term in the cross section which is independent of azimuthal angle [15]. For instance, Higgs boson and quarkonium productions do not require any angular analysis to extract $h_{1}^{\perp g}$. The Higgs $p_{T}$ distribution can be modified by taking linearly polarized gluons into account in unpolarized $p p$ collision at LHC $[15,16,17]$. Whether the Higgs is a scalar or pseudo scalar can be understood through the modified $p_{T}$ spectrum. 
It is also suggested that Higgs+jet production in $p p$ collision at LHC [18] is helpful to probe $h_{1}^{\perp g}$. Quarkonium production (even charge conjugation) has been studied in non-relativistic Quantum Chromodynamics (NRQCD) version of color singlet model (CSM) to investigate $h_{1}^{\perp g}$ [19].

$J / \psi$ and $\Upsilon(1 \mathrm{~S})$ bound states have been of interest, not only because it is possible to measure the production experimentally but also it gives information on the strong interaction responsible for hadronization. In the earlier work [20], we proposed $J / \psi$ and $\Upsilon(1 \mathrm{~S})$ production in $p p$ collision to explore the effect of linearly polarized gluons using color evaporation model (CEM). The present article discusses about probing $f_{1}^{g}$ and $h_{1}^{\perp g}$ in quarkonium $(J / \psi$ and $\Upsilon(1 \mathrm{~S}))$ production through $g g$ fusion channel at leading order (LO) in $p p$ collision using color octet model (COM) in TMD factorization framework.

CSM, CEM and COM are three notable models for quarkonium production which are successful at different energies. More recently, in Ref. [21, 22], color glass condensate model (CGS) and NRQCD NLO framework have been used to explain the full $p_{T}$ spectrum of $J / \psi$ in protonproton and proton-nucleus collision at RHIC. All these models are based on a common assumption : that the production rate is factorized into a product of short and long distance factors. The production of $Q \bar{Q}$ pair calculated through perturbation theory, depends on specific process like $e p$ or $p p$. The produced $Q \bar{Q}$ pair transmutes into physical color singlet meson by radiating soft gluons. This process can not be calculated using perturbative QCD tools. Moreover, there are two scales $[23,24]$ involved in the quarkonium production. The $Q \bar{Q}$ pair production takes place within a short time of order $1 / M_{Q \bar{Q}}$, where $M_{Q \bar{Q}}$ is the mass of quarkonium. The binding process happens at a long time of order $1 / \Lambda_{Q C D}[23,24]$.

CSM model was developed by E. L. Berger and D. Jones $[25,26,27]$ after the discovery of $J / \psi$ $[28,29]$. In CSM, the heavy quark pair is produced in color singlet state with certain spin $(\mathrm{S})$ and orbital angular quantum number (L) and later it evolves into quarkonium, nonrelativistic bound state. The long distance factor (wave function or its derivative) contains the nonperturbative information of quarkonium. Generally, these objects are obtained from potential models or experimental data. CSM was able to predict the quarkonium production at low energy [30] but at high energy it requires large corrections in $\alpha_{s}[31,32]$. In CEM model, as introduced by F.Halzen, Matsuda [33, 34] and Fritsch [35], the production rate is product of a long distance 
factor and the cross section of heavy quark pair integrated over the invariant mass of the $Q \bar{Q}$ pair below the threshold mass. In CEM, it is assumed that the probability of producing the quarkonium state is independent of color and spin quantum number of initial heavy quark pair. It implies that the color of initial $Q \bar{Q}$ pair does not play any role in hadronization process. It was successful in describing $p_{T}$ distribution of $J / \psi, \psi(2 S)$ and $\chi$ at $\sqrt{s}=1.8 \mathrm{TeV}$ (CDF) $[23,24]$.

Bodwin, Braaten and Lepage [36] proposed COM based on NRQCD effective field theory. It is assumed in COM that the heavy quark relative momentum $\left(M_{Q} v\right)$ is much less than the mass $\left(M_{Q}\right)$ of heavy quark, where $v$ is the relative velocity of heavy quark in the rest frame of quarkonium. The ignored relativistic corrections in CSM are included in COM. The cross section of quarkonium is expressed as an infinite series in the limit $v \ll 1$. Each term

in the series is the product of $Q \bar{Q}$ pair cross section in a definite state $n={ }^{2 S+1} L_{J}^{[a]}$ and long distance matrix elements (LDME). Here, $J, L$ and $S$ are total angular momentum, orbital angular momentum and spin quantum numbers respectively. $a$ is the color multiplicity bearing a value of 1 for color singlet and 8 for color octet state. In this model, both the color singlet and color octet states are included in the production rate of quarkonium. The infinite series can be truncated for practical purpose. LDME describes the transition probability to form the quarkonium state from the heavy quark pair and is extracted from experimental data. The COM gives a good description of $J / \psi$ at RHIC energy [37]. The present paper employs the NRQCD based model to study the TMDs and their evolution. Moreover, we compare the $J / \psi$ and $\Upsilon(1 \mathrm{~S})$ production obtained in COM with earlier work which implemented CEM [20]. The paper is organized as the following. Quarkonium production is presented in Sec.II and Sec.III describes the TMD evolution formalism. Numerical results and conclusions are presented in Sec.IV and Sec.V respectively.

\section{QUARKONIUM PRODUCTION}

NRQCD formalism provides a theoretical framework to calculate the cross section of bound states. As per NRQCD, the differential cross section of any quarkonium state is factorized as 
the following $[38,39]$

$$
d \sigma^{J / \psi(\Upsilon(1 \mathrm{~S}))}=\sum_{n} d \hat{\sigma}[a b \rightarrow Q \bar{Q}(n)]\left\langle 0\left|\mathcal{O}_{n}^{J / \psi(\Upsilon(1 \mathrm{~S}))}\right| 0\right\rangle
$$

where $d \hat{\sigma}$ is the partonic level differential cross section of $Q \bar{Q}$ pair calculated in order $\alpha_{s}$. The color, spin and angular momentum quantum numbers are collectively denoted with $n$. Here, $a$ and $b$ can be gluons and quarks. The $Q(\bar{Q})$ is a heavy quark (heavy anti-quark). The LDME, $\left\langle 0\left|\mathcal{O}_{n}^{J / \psi(\Upsilon(1 \mathrm{~S}))}\right| 0\right\rangle$, contains the nonperturbative effects like hadronization of $Q \bar{Q}$ pair which is defined as a four fermion operator [38]. We consider the unpolarized $p p$ collision process for the charmonium and bottomonium production

$$
P\left(p_{A}\right)+P\left(p_{B}\right) \rightarrow J / \psi \text { or } \Upsilon(1 \mathrm{~S})(p)+X
$$

the four momenta are given within round brackets. We consider a frame where the colliding protons are moving along the $+\hat{z}$ axis and $-\hat{z}$ axis. The four momenta in the center of mass frame are $P_{A}^{\mu}=\frac{\sqrt{s}}{2}(1,0,0,1)$ and $P_{B}^{\mu}=\frac{\sqrt{s}}{2}(1,0,0,-1)$. The leading channel is gluon-gluon collision since proton is rich of gluons at high energy. Therefore we consider the leading order (LO) gluon fusion subprocess for the quarkonium production, i.e., $g g \rightarrow Q \bar{Q}\left[{ }^{2 s+1} L_{J}^{(a)}\right]$. In line with Ref. [19, 40], we assume that the QCD factorization theorem can be applicable at high energy to separate out the short and long distance effects systematically. Using TMD factorization and NRQCD formalism, the differential cross section of the quarkonium is given by

$$
d \sigma=\int d x_{a} d x_{b} d^{2} \mathbf{k}_{\perp a} d^{2} \mathbf{k}_{\perp b} \Phi_{g}^{\mu \nu}\left(x_{a}, \mathbf{k}_{\perp a}\right) \Phi_{g \mu \nu}\left(x_{b}, \mathbf{k}_{\perp b}\right) d \sigma^{J / \psi(\Upsilon(1 \mathrm{~S}))}
$$

where $\Phi_{g}^{\mu \nu}\left(x_{a}, \mathbf{k}_{\perp a}\right)$ is the gluon correlator of the spin- $\frac{1}{2}$ unpolarized proton which is parametrized in terms of leading twist traditional TMDs [10] as following

$$
\Phi_{g}^{\mu \nu}\left(x, \mathbf{k}_{\perp}\right)=-\frac{1}{2 x}\left\{g_{T}^{\mu \nu} f_{1}^{g}\left(x, \mathbf{k}_{\perp}^{2}\right)-\left(\frac{k_{\perp}^{\mu} k_{\perp}^{\nu}}{M_{h}^{2}}+g_{T}^{\mu \nu} \frac{\mathbf{k}_{\perp}^{2}}{2 M_{h}^{2}}\right) h_{1}^{\perp g}\left(x, \mathbf{k}_{\perp}^{2}\right)\right\} .
$$

Here, $x$ and $k_{\perp}$ represent the longitudinal momentum fraction and transverse momentum of the gluon respectively. $M_{h}$ is the mass of the proton. $f_{1}^{g}\left(x, \mathbf{k}_{\perp}^{2}\right)$ describes the density of unpolarized gluons and linearly polarized gluon distribution is represented by $h_{1}^{\perp g}\left(x, \mathbf{k}_{\perp}^{2}\right)$ inside an unpolarized proton. 
The partonic differential cross section is given by [38]

$$
\begin{aligned}
d \sigma^{J / \psi(\Upsilon(1 \mathrm{~S}))=} & \frac{5 \alpha_{s}^{2} \pi^{3}}{96 m_{Q}^{3}} \frac{1}{2} d^{2} \mathbf{p}_{T} d y \delta^{4}\left(p_{a}+p_{b}-p\right)\left\{\left\langle 0\left|\mathcal{O}_{8}^{J / \psi(\Upsilon(1 \mathrm{~S}))}\left({ }^{1} S_{0}\right)\right| 0\right\rangle\right. \\
& \left.+\frac{3}{m_{Q}^{2}}\left\langle 0\left|\mathcal{O}_{8}^{J / \psi(\Upsilon(1 \mathrm{~S}))}\left({ }^{3} P_{0}\right)\right| 0\right\rangle+\frac{4}{5 m_{Q}^{2}}\left\langle 0\left|\mathcal{O}_{8}^{J / \psi(\Upsilon(1 \mathrm{~S}))}\left({ }^{3} P_{2}\right)\right| 0\right\rangle\right\} \\
= & C_{n} d^{2} \mathbf{p}_{T} d y \delta^{4}\left(p_{a}+p_{b}-p\right),
\end{aligned}
$$

where, the four momentum vectors of the incoming gluons in center of mass frame are denoted with $p_{a}=x_{a} \frac{\sqrt{s}}{2}\left(1+\frac{k_{\perp a}^{2}}{x_{a}^{2} s}, \frac{2 \mathbf{k}_{\perp a}}{x_{a} \sqrt{s}}, 1-\frac{k_{\perp a}^{2}}{x_{a}^{2} s}\right)$ and $p_{b}=x_{b} \frac{\sqrt{s}}{2}\left(1+\frac{k_{\perp b}^{2}}{x_{b}^{2} s}, \frac{2 \mathbf{k}_{\perp b}}{x_{b} \sqrt{s}},-1+\frac{k_{\perp b}^{2}}{x_{b}^{2} s}\right)$ [41]. The $Q \bar{Q}$ pair four momentum is $p=\left(p_{0}, \mathbf{p}_{T}, p_{L}\right)$. Here, $m_{Q}$ is mass of heavy quark. $\mathbf{p}_{T}$ and $y$ are the transverse momentum and rapidity of the quarkonium respectively. $C_{n}$ is defined as the following

$$
\begin{aligned}
C_{n}= & \frac{5 \alpha_{s}^{2} \pi^{3}}{96 m_{Q}^{3}} \frac{1}{2}\left\{\left\langle 0\left|\mathcal{O}_{8}^{J / \psi(\Upsilon(1 \mathrm{~S}))}\left({ }^{1} S_{0}\right)\right| 0\right\rangle+\frac{3}{m_{Q}^{2}}\left\langle 0\left|\mathcal{O}_{8}^{J / \psi(\Upsilon(1 \mathrm{~S}))}\left({ }^{3} P_{0}\right)\right| 0\right\rangle\right. \\
& \left.+\frac{4}{5 m_{Q}^{2}}\left\langle 0\left|\mathcal{O}_{8}^{J / \psi(\Upsilon(1 \mathrm{~S}))}\left({ }^{3} P_{2}\right)\right| 0\right\rangle\right\} .
\end{aligned}
$$

As per Ref. [37, 38], the color octet states ${ }^{1} S_{0},{ }^{3} P_{0}$ and ${ }^{3} P_{2}$ contribution is dominant for charmonium and bottomonium production in $g g \rightarrow Q \bar{Q}\left[{ }^{2 S+1} L_{J}^{(a)}\right]$ subprocess. The scattering amplitudes of ${ }^{3} S_{1}$ and ${ }^{3} P_{1}$ states vanish when two initial scattering gluons are on-shell gluons $[38,42,43]$. We consider two sets ("Set-I" and "Set-II") of LDMEs for quarkonium production. In Set-I, the numerical values of LDME for $J / \psi[22,44]$ and $\Upsilon(1 \mathrm{~S})$ [45] are extracted by fitting $J / \psi$ data at Tevatron and $\Upsilon(1 \mathrm{~S})$ production at LHC in NLO collinear factorization using NRQCD framework. For Set-II, LDMEs are taken from Ref. [46, 47] and [48] for $J / \psi$ and $\Upsilon(1 \mathrm{~S})$ respectively. Feeddown contributions from $\chi_{c}$ and $\psi(2 s)$ were included for extracting LDMEs of $J / \psi$ in Set-II unlike in Set-I. LDME numerical values are given tabular form in TABLE I. The numerical value of LDME for $J=2$ state is obtained by using Eq.(6.6) from Ref. [39]. The differential cross section in terms of TMDs is obtained by substituting Eq.(4) and (5) in Eq.(3)

$$
\begin{aligned}
\frac{d^{4} \sigma}{d y d^{2} \mathbf{p}_{T}}= & \frac{C_{n}}{2} \int \frac{d x_{a}}{x_{a}} \frac{d x_{b}}{x_{b}} d^{2} \mathbf{k}_{\perp a} d^{2} \mathbf{k}_{\perp b} \delta^{4}\left(p_{a}+p_{b}-p\right) \\
& \times\left[f_{1}^{g}\left(x_{a}, \mathbf{k}_{\perp a}^{2}\right) f_{1}^{g}\left(x_{b}, \mathbf{k}_{\perp b}^{2}\right)+w h_{1}^{\perp g}\left(x_{a}, \mathbf{k}_{\perp a}^{2}\right) h_{1}^{\perp g}\left(x_{b}, \mathbf{k}_{\perp b}^{2}\right)\right]
\end{aligned}
$$


TABLE I. Numerical values of LDME.

\begin{tabular}{ccc}
\hline \hline$\left\langle 0\left|\mathcal{O}_{8}^{J / \psi(\Upsilon(1 \mathrm{~S}))}\left({ }^{2 S+1} L_{J}\right)\right| 0\right\rangle$ & Set-I $[22,44,45]$ & Set-II $[46,47,48]$ \\
\hline$\left\langle 0\left|\mathcal{O}_{8}^{J / \psi}\left({ }^{1} S_{0}\right)\right| 0\right\rangle / \mathrm{GeV}^{3}$ & 0.089 & 0.097 \\
$\left\langle 0\left|\mathcal{O}_{8}^{J / \psi}\left({ }^{3} P_{0}\right)\right| 0\right\rangle / \mathrm{GeV}^{5}$ & 0.0126 & -0.0214 \\
$\left\langle 0\left|\mathcal{O}_{8}^{J / \psi}\left({ }^{3} P_{2}\right)\right| 0\right\rangle / \mathrm{GeV}^{5}$ & 0.063 & -0.107 \\
$\left\langle 0\left|\mathcal{O}_{8}^{\Upsilon(1 \mathrm{~S})}\left({ }^{1} S_{0}\right)\right| 0\right\rangle / \mathrm{GeV}^{3}$ & 0.0121 & 0.111 \\
$\left\langle 0\left|\mathcal{O}_{8}^{\Upsilon(1 \mathrm{~S})}\left({ }^{3} P_{0}\right)\right| 0\right\rangle / \mathrm{GeV}^{5}$ & 1.440 & -0.151 \\
$\left\langle 0\left|\mathcal{O}_{8}^{\Upsilon(1 \mathrm{~S})}\left({ }^{3} P_{2}\right)\right| 0\right\rangle / \mathrm{GeV}^{5}$ & 7.203 & -0.755 \\
\hline \hline
\end{tabular}

where $w$ is the transverse momentum weight factor

$$
w=\frac{1}{2 M_{h}^{4}}\left[\left(\mathbf{k}_{\perp a} \cdot \mathbf{k}_{\perp b}\right)^{2}-\frac{1}{2} \mathbf{k}_{\perp a}^{2} \mathbf{k}_{\perp b}^{2}\right] .
$$

The four momentum conservation delta function can be written as [41]

$$
\begin{aligned}
\delta^{4}\left(p_{a}+p_{b}-q\right) & =\delta\left(E_{a}+E_{b}-p_{0}\right) \delta\left(p_{z a}+p_{z b}-p_{L}\right) \delta^{2}\left(\mathbf{k}_{\perp a}+\mathbf{k}_{\perp b}-\mathbf{p}_{T}\right) \\
& =\frac{2}{s} \delta\left(x_{a}-\frac{M e^{y}}{\sqrt{s}}\right) \delta\left(x_{b}-\frac{M e^{-y}}{\sqrt{s}}\right) \delta^{2}\left(\mathbf{k}_{\perp a}+\mathbf{k}_{\perp b}-\mathbf{p}_{T}\right),
\end{aligned}
$$

where, $M$ is the mass of quarkonium. After integrating with respect to $x_{a}$ and $x_{b}$, the two $\delta$ functions contained in the above equation gives

$$
x_{a, b}=\frac{M}{\sqrt{s}} e^{ \pm y}
$$

We can also eliminate $\mathbf{k}_{\perp b}$ by integrating and we get

$$
\frac{d \sigma^{f f+h h}}{d y d^{2} \mathbf{p}_{T}}=\frac{d \sigma^{f f}}{d y d^{2} \mathbf{p}_{T}}+\frac{d \sigma^{h h}}{d y d^{2} \mathbf{p}_{T}}
$$

where

$$
\frac{d \sigma^{f f}}{d y d^{2} \mathbf{p}_{T}}=\frac{C_{n}}{s} \int d^{2} \mathbf{k}_{\perp a} f_{1}^{g}\left(x_{a}, \mathbf{k}_{\perp a}^{2}\right) f_{1}^{g}\left(x_{b},\left(\mathbf{p}_{T}-\mathbf{k}_{\perp a}\right)^{2}\right)
$$

and

$$
\begin{aligned}
\frac{d \sigma^{h h}}{d y d^{2} \mathbf{p}_{T}}= & \frac{C_{n}}{s} \frac{1}{2 M_{h}^{4}} \int d^{2} \mathbf{k}_{\perp a}\left[\left(\mathbf{k}_{\perp a} \cdot\left(\mathbf{p}_{T}-\mathbf{k}_{\perp a}\right)\right)^{2}-\frac{1}{2} \mathbf{k}_{\perp a}^{2}\left(\mathbf{p}_{T}-\mathbf{k}_{\perp a}\right)^{2}\right] \\
& \times h_{1}^{\perp g}\left(x_{a}, \mathbf{k}_{\perp a}^{2}\right) h_{1}^{\perp g}\left(x_{b},\left(\mathbf{p}_{T}-\mathbf{k}_{\perp a}\right)^{2}\right)
\end{aligned}
$$




\section{TMD EVOLUTION}

In this section, Dokshitzer-Gribov-Lipatov-Altarelli-Parisi (DGLAP) and TMD evolutions are discussed. Generally, we assume that the unpolarized gluon TMDs exhibit Gaussian distribution. The widely used Gaussian parametrization of TMDs is given by

$$
f_{1}^{g}\left(x, \mathbf{k}_{\perp}^{2}\right)=f_{1}^{g}\left(x, Q^{2}\right) \frac{1}{\pi\left\langle k_{\perp}^{2}\right\rangle} e^{-\mathbf{k}_{\perp}^{2} /\left\langle k_{\perp}^{2}\right\rangle}
$$

Here, we factorized the TMD PDF into $x$ and $k_{\perp}$ dependencies. $f_{1}^{g}\left(x, Q^{2}\right)$ is the collinear PDF measured at the probing scale $Q^{2}=M^{2}$ and scale evolution in the $k_{\perp}$ dependent term is not taken into consideration. This is called the DGLAP evolution approach. The Gaussian form of $h_{1}^{\perp g}$ is the following [19]

$$
h_{1}^{\perp g}\left(x, \mathbf{k}_{\perp}^{2}\right)=\frac{M_{h}^{2} f_{1}^{g}\left(x, Q^{2}\right)}{\pi\left\langle k_{\perp}^{2}\right\rangle^{2}} \frac{2(1-r)}{r} e^{1-\mathbf{k}_{\perp}^{2} \frac{1}{r\left\langle k_{\perp}^{2}\right\rangle}}
$$

where, $r(0<r<1)$ is the parameter. We take $\left\langle k_{\perp}^{2}\right\rangle=0.25 \mathrm{GeV}^{2}$ and $1 \mathrm{GeV}^{2}$ [19] and $r=\frac{1}{3}$ and $\frac{2}{3}$ [19] for numerical calculation. Model independent upper bound for $h_{1}^{\perp g}$ is given in [11] and is obeyed by the Eq.(16) for all values of $k_{\perp}$ and $x$

$$
\frac{\mathbf{k}_{\perp}^{2}}{2 M_{h}^{2}}\left|h_{1}^{\perp}\left(x, \mathbf{k}_{\perp}^{2}\right)\right| \leq f_{1}^{g}\left(x, \mathbf{k}_{\perp}^{2}\right) .
$$

\section{A. Model-I}

In model-I, an upper limit of transverse momentum integration is not chosen. The Gaussian form of the unpolarized and linearly polarized TMDs allow us to integrate analytically and we get

$$
\frac{d^{2} \sigma^{f f}}{d y d p_{T}^{2}}=\frac{C_{n} \beta}{2 s} e^{-\frac{\beta p_{T}^{2}}{2}} f_{1}^{g}\left(x_{a}\right) f_{1}^{g}\left(x_{b}\right)
$$

and

$$
\frac{d^{2} \sigma^{h h}}{d y d p_{T}^{2}}=\frac{C_{n} \beta(1-r)^{2} r e^{2}}{4 s} e^{-\frac{\beta p_{T}^{2}}{2 r}}\left[1-\frac{\beta p_{T}^{2}}{r}+\frac{\beta^{2} p_{T}^{4}}{8 r^{2}}\right] f_{1}^{g}\left(x_{a}\right) f_{1}^{g}\left(x_{b}\right) .
$$

where $\beta=\frac{1}{\left\langle k_{\perp a}^{2}\right\rangle}=\frac{1}{\left\langle\left(p_{T}-k_{\perp a}\right)^{2}\right\rangle}$. 


\section{B. Model II}

In model-II, for Gaussian distribution functions the effective intrinsic motion of partons is limited to $k_{\max }=\sqrt{\left\langle k_{\perp a}^{2}\right\rangle}$ [49]. The final expressions we have

$$
\frac{d^{2} \sigma^{f f}}{d y d p_{T}^{2}}=\frac{C_{n} \beta^{2}}{2 s \pi^{2}} \int_{0}^{2 \pi} d \phi_{p_{T}} \int_{0}^{2 \pi} d \phi_{k_{\perp a}} \int_{0}^{\sqrt{\left\langle k_{\perp a}^{2}\right\rangle}} k_{\perp a} d k_{\perp a} e^{-\beta \Delta} f_{1}^{g}\left(x_{a}\right) f_{1}^{g}\left(x_{b}\right),
$$

and

$$
\begin{aligned}
\frac{d^{2} \sigma^{h h}}{d y d p_{T}^{2}}= & \frac{C_{n} \beta^{4}}{s \pi^{2}} \frac{(1-r)^{2} e^{2}}{r^{2}} \int_{0}^{2 \pi} d \phi_{p_{T}} \int_{0}^{2 \pi} d \phi_{k_{\perp a}} \int_{0}^{\sqrt{\left\langle k_{\perp a}^{2}\right\rangle}} k_{\perp a} d k_{\perp a}\left[\frac{1}{2} k_{\perp a}^{4}-\frac{1}{2} k_{\perp a}^{2} p_{T}^{2}\right. \\
& \left.-p_{T} k_{\perp a}^{3} \cos \left(\phi_{k_{\perp a}}-\phi_{p_{T}}\right)+p_{T}^{2} k_{\perp a}^{2} \cos ^{2}\left(\phi_{k_{\perp a}}-\phi_{p_{T}}\right)\right] e^{-\frac{\beta}{r} \Delta} f_{1}^{g}\left(x_{a}\right) f_{1}^{g}\left(x_{b}\right) .
\end{aligned}
$$

where $\Delta=2 k_{\perp a}^{2}+p_{T}^{2}-2 p_{T} k_{\perp a} \cos \left(\phi_{k_{\perp a}}-\phi_{p_{T}}\right)$. The azimuthal angle of gluon and quarkonium are $\phi_{k_{\perp a}}$ and $\phi_{p_{T}}$ respectively. We have chosen $\left\langle k_{\perp a}^{2}\right\rangle=\left\langle k_{\perp}^{2}\right\rangle$ for numerical estimation of quarkonium production rate. DGLAP evolution approach could not describe the Z-boson high transverse momentum distribution in DY process at CDF [8]. Nevertheless, one has to consider TMD evolution approach to explain high $p_{T}$ data [8]. In TMD evolution approach, we follow the formalism adopted in Ref. [20] to study scale evolution of TMDs. The quarkonium differential cross section in $b_{\perp}$-space is derived by following Eq.(29) to Eq.(33) from Ref. [20]

$$
\frac{d \sigma}{d y d^{2} \mathbf{p}_{T}}=\frac{C_{n}}{s} \frac{1}{2 \pi} \int_{0}^{\infty} b_{\perp} d b_{\perp} J_{0}\left(p_{T} b_{\perp}\right)\left\{f_{1}^{g}\left(x_{a}, b_{\perp}^{2}\right) f_{1}^{g}\left(x_{b}, b_{\perp}^{2}\right)+h_{1}^{\perp g}\left(x_{a}, b_{\perp}^{2}\right) h_{1}^{\perp g}\left(x_{b}, b_{\perp}^{2}\right)\right\},
$$

where $J_{0}$ is the zeroth order Bessel function. In TMD factorization theorem, spurious light cone divergences appear [1] which can be regularized by introducing auxiliary parameter $\zeta$. As a result, TMDs depend on renormalization scale $\mu$ and $\zeta$. Using CS and RG equations [1, 50] one can evolve the TMDs from initial scale $Q_{i}=c / b_{*}\left(b_{\perp}\right)=\zeta_{0}$ to final scale $Q_{f}=Q=\zeta[50,51]$

$$
f\left(x, b_{\perp}, Q_{f}, \zeta\right)=f\left(x, b_{\perp}, Q_{i}, \zeta\right) R_{\text {pert }}\left(Q_{f}, Q_{i}, b_{*}\right) R_{N P}\left(Q_{f}, Q_{i}, b_{\perp}\right)
$$

where $R_{\text {pert }}$ is the perturbative part of the evolution kernel and can be calculated using perturbation theory. $R_{N P}$ is the nonperturbative part of evolution kernel and TMDs. Initial scale of TMDs is chosen to be $Q_{i}=c / b_{*}$, where $c=2 e^{-\gamma_{\epsilon}}$ with $\gamma_{\epsilon} \approx 0.577$. The initial scale

$Q_{i}$ becomes small when $b_{\perp}$ is large, as a result we enter in the nonperturbative regime [1]. The $b_{*}$ prescription is adopted to separate the evolution kernel nonperturbative part, where 
$b_{*}\left(b_{\perp}\right)=\frac{b_{\perp}}{\sqrt{1+\left(\frac{b_{\perp}}{b_{\max }}\right)^{2}}} \approx b_{\max }$ when $b_{\perp} \rightarrow \infty$ and $b_{*}\left(b_{\perp}\right) \approx b_{\perp}$ when $b_{\perp} \rightarrow 0$. $R_{N P}$ contains nonperturbative information of evolution kernel that cannot be calculated and need to be parametrized. The perturbative evolution kernel is given by [52]

$$
R_{\text {pert }}\left(Q_{f}, Q_{i}, b_{*}\right)=\exp \left\{-\int_{c / b_{*}}^{Q} \frac{d \mu}{\mu}\left(A \log \left(\frac{Q^{2}}{\mu^{2}}\right)+B\right)\right\}
$$

where the anomalous dimensions are denoted by $A$ an $B$ respectively and these have perturbative expansion as follows :

$$
A=\sum_{n=1}^{\infty}\left(\frac{\alpha_{s}(\mu)}{\pi}\right)^{n} A_{n}
$$

and

$$
B=\sum_{n=1}^{\infty}\left(\frac{\alpha_{s}(\mu)}{\pi}\right)^{n} B_{n}
$$

$A_{1}=C_{A}$ and $B_{1}=-\frac{1}{2}\left(\frac{11}{3} C_{A}-\frac{2}{3} N_{f}\right)$ are the anomalous dimension coefficients of order in $\alpha_{s}$. The anomalous dimensions have been calculated up to 3-loop level [53]. The evolution kernel is the same for linearly polarized gluons since it is independent of type of TMDs. As stated before, the non-perturbative part of the evolution kernel cannot be calculated, and a parametrized form has to be chosen. Here we use two nonperturbative factor parametrizations which are called "AR" and "BLNY". The "AR" nonperturbative Sudakov factor was cansidered by Aybat and Rogers [50] and is successful in describing the low energy SIDIS and DY data

$$
R_{N P}\left(x, Q, b_{\perp}\right)=\exp \left\{-\left[\frac{g_{2}}{2} \log \frac{Q}{2 Q_{0}}+\frac{g_{1}}{2}\left(1+2 g_{3} \log \frac{10 x x_{0}}{x_{0}+x}\right)\right] b_{\perp}^{2}\right\} .
$$

The "BLNY" Sudakov nonperturbative factor was used by Sun et al. [54] in quarkonium production and is given by

$$
R_{N P}\left(x, Q, b_{\perp}\right)=\exp \left\{-\left[\frac{g_{2}}{2} \log \frac{Q}{2 Q_{0}}+\frac{g_{1}}{2}+g_{1} g_{3} \log (10 x)\right] b_{\perp}^{2}\right\} .
$$

The numerical values of the best fit parameters are given in TABLE II. Though, $R_{N P}$ is $x$ dependent, we choose $x_{a}=x_{b}=0.09$ only for "AR" $R_{N P}$ as per Ref. $[50,52]$ to write the $R_{N P}$ in the form of a Gaussian function. For "BLNY" $R_{N P}$, Eq.(11) is used for $x_{a}$ and $x_{b}$. We choose Eq.(25) and (26) as the nonperturbative Sudakov factors for linearly polarized gluon TMD PDF as well since no experimental data is available to extract the best fit parameters of 


\begin{tabular}{|c|c|c|c|c|c|c|}
\hline$R_{N P}$ & $g_{1} / \mathrm{GeV}^{2}$ & $g_{2} / \mathrm{GeV}^{2}$ & $g_{3}$ & $Q_{0} / \mathrm{GeV}$ & $\mathrm{b}_{\max } / \mathrm{GeV}^{-1}$ & $x_{0}$ \\
\hline $\mathrm{AR}[50]$ & 0.201 & 0.184 & -0.129 & 1.6 & 1.5 & 0.009 \\
\hline BLNY [54] & 0.03 & 0.87 & -5.66 & 1.6 & 0.5 & \\
\hline
\end{tabular}

TABLE II. Best fit parameters of nonperturbative Sudakov factor $\left(R_{N P}\right)$

$R_{N P}$ for $h_{1}^{\perp g}$. In general, TMDs are written as the convolution of coefficient function times the collinear PDF

$$
f\left(x, b_{\perp}, Q_{i}, \zeta\right)=\sum_{i=g, q} \int_{x}^{1} \frac{d \hat{x}}{\hat{x}} C_{i / g}\left(x / \hat{x}, b_{\perp}, \alpha_{s}, Q_{i}, \zeta\right) f_{i / p}\left(\hat{x}, c / b_{*}\right)+\mathcal{O}\left(b_{\perp} \Lambda_{Q C D}\right),
$$

where the coefficient function is dependent on the type of TMD and is independent of the process, this is calculated using perturbation theory. The unpolarized and linearly polarized TMDs in terms of collinear PDFs at leading and first order in $\alpha_{s}$ are [52]

$$
\begin{gathered}
f_{1}^{g}\left(x, b_{\perp}, Q_{i}, \zeta\right)=f_{g / p}\left(x, c / b_{*}\right)+\mathcal{O}\left(\alpha_{s}\right) \\
h_{1}^{\perp g}\left(x, b_{\perp}, Q_{i}, \zeta\right)=\frac{\alpha_{s}\left(c / b_{*}\right) C_{A}}{\pi} \int_{x}^{1} \frac{d \hat{x}}{\hat{x}}\left(\frac{\hat{x}}{x}-1\right) f_{g / p}\left(\hat{x}, c / b_{*}\right)+\mathcal{O}\left(\alpha_{s}^{2}\right) .
\end{gathered}
$$

Using above equations one can rewrite Eq.(22) as

$$
\frac{d^{2} \sigma^{f f+h h}}{d y d p_{T}^{2}}=\frac{d^{2} \sigma^{f f}}{d y d p_{T}^{2}}+\frac{d^{2} \sigma^{h h}}{d y d p_{T}^{2}}
$$

where

$$
\begin{aligned}
\frac{d^{2} \sigma^{f f}}{d y d p_{T}^{2}}=\frac{C_{n}}{2 s} \int_{0}^{\infty} b_{\perp} d b_{\perp} J_{0}\left(p_{T} b_{\perp}\right) f_{1}^{g}\left(x_{a}, c / b_{*}\right) f_{1}^{g}\left(x_{b}, c / b_{*}\right) \exp \left\{-2 \int_{c / b_{*}}^{Q} \frac{d \mu}{\mu}\left(A \log \left(\frac{Q^{2}}{\mu^{2}}\right)+B\right)\right\} \\
\times R_{N P}\left(x_{a}, Q, b_{\perp}\right) R_{N P}\left(x_{b}, Q, b_{\perp}\right),
\end{aligned}
$$

and

$$
\begin{aligned}
\frac{d^{2} \sigma^{h h}}{d y d p_{T}^{2}}= & \frac{C_{n} C_{A}^{2}}{2 s \pi^{2}} \int_{0}^{\infty} b_{\perp} d b_{\perp} J_{0}\left(p_{T} b_{\perp}\right) \alpha_{s}^{2}\left(c / b_{*}\right) \int_{x_{a}}^{1} \frac{d x_{1}}{x_{1}}\left(\frac{x_{1}}{x_{a}}-1\right) f_{1}^{g}\left(x_{1}, c / b_{*}\right) \int_{x_{b}}^{1} \frac{d x_{2}}{x_{2}}\left(\frac{x_{2}}{x_{b}}-1\right) \\
& f_{1}^{g}\left(x_{2}, c / b_{*}\right) \exp \left\{-2 \int_{c / b_{*}}^{Q} \frac{d \mu}{\mu}\left(A \log \left(\frac{Q^{2}}{\mu^{2}}\right)+B\right)\right\} R_{N P}\left(x_{a}, Q, b_{\perp}\right) R_{N P}\left(x_{b}, Q, b_{\perp}\right) .
\end{aligned}
$$




\section{NUMERICAL RESULTS}

MSTW2008 is used for numerical calculations [55]. Masses of $J / \psi$ and $\Upsilon(1 \mathrm{~S})$ are taken as $M=3.096$ and $9.398 \mathrm{GeV}$ respectively. The transverse momentum $\left(p_{T}\right)$ and rapidity $(y)$ distributions of $J / \psi$ and $\Upsilon(1 \mathrm{~S})$ are estimated in unpolarized $p p$ collision at $\sqrt{s}=7 \mathrm{TeV}(\mathrm{LHCb})$, $\sqrt{s}=500 \mathrm{GeV}$ (RHIC) and $\sqrt{s}=115 \mathrm{GeV}$ (AFTER) in NRQCD formalism using TMD factorization. We have considered the color octet states in LO subprocess $g g \rightarrow Q \bar{Q}\left[{ }^{2 s+1} L_{J}^{8}\right]$ for quarkonium production. To obtain the cross section differential in $p_{T}$, the integration of rapidity is chosen in the range of $y \in[2.0,4.5], y \in[-3.0,3.0]$ and $y \in[-0.5,0.5]$ for LHCb, RHIC and AFTER respectively. In general, the LDME $\left(\left\langle 0\left|\mathcal{O}_{n}^{J / \psi(\Upsilon(1 \mathrm{~S}))}\right| 0\right\rangle\right)$ in COM and $\rho$ in CEM depend on the mass of heavy quark $\left(m_{Q}\right)$, scale $Q$, order of the calculation (LO, NLO) and PDFs $f\left(x, Q^{2}\right)[56]$. Hence, $m_{c}=1.5 \mathrm{GeV}$ and $m_{b}=4.88 \mathrm{GeV}$ in COM is chosen in line with Ref. [22] and [45] respectively. In CEM, $m_{c}=1.2 \mathrm{GeV}$ and $m_{b}=4.75 \mathrm{GeV}$ is considered $[20,57]$ for charm and bottom quark masses.

In all the figures, the conventions are the following. "ff" represents the distribution of quarkonium and is obtained by taking into account only the unpolarized gluon contribution in the scattering process. The distribution of quarkonium denoted with "ff+hh" is obtained by considering both unpolarized and linearly polarized gluons in $p p$ collision. Two sets of LDMEs i.e., "Set-I" and "Set-II" are considered for color octet states which are given in TABLE I. The $p_{T}$ and $y$ spectra of quarkonium are estimated both in DGLAP and TMD evolution approach.

The transverse momentum distribution is evaluated in model-I from Eq.(18) and Eq.(19) and in model-II from Eq.(20) and Eq.(21). We have taken two values of the Gaussian width $\left\langle k_{\perp}^{2}\right\rangle=0.25,1 \mathrm{GeV}^{2}$ and two values for the parameter $r=\frac{1}{3}, \frac{2}{3}$ for the numerical estimation of $p_{T}$ and $y$ spectra of quarkonium in DGLAP evolution approach. In FIG.1-12, "Set-I" LDMEs and "AR" $R_{N P}$ factor are used for color octet states and TMD evolution respectively. $p_{T}$ spectrum of quarkonium is normalized with total cross section in FIG.1 and FIG.2 in model-I and model-II resulting in the cancellation of scale dependent terms. As a result, $p_{T}$ spectrum of quarkonium is independent of center of mass energy and quarkonium mass as shown in FIG.1 and FIG.2. The quarkonium $p_{T}$ spectra presented in FIG.1 and FIG.2 agree with that we obtained in Ref. [20] using CEM. The contribution of linearly polarized gluons in $p_{T}$ integrated 
cross section is zero. Noticeable modifications in the quarkonium $p_{T}$ distribution are observed upon taking the linearly polarized gluons into consideration along with the unpolarized gluons, in the scattering process. The effect of linearly polarized gluons on the $p_{T}$ spectrum of $J / \psi$ and $\Upsilon(1 \mathrm{~S})$ is limited to low $p_{T}<0.5 \mathrm{GeV}$. Model II gives higher values of the normalized cross section compared to model I.

Rapidity distribution of $J / \psi$ and $\Upsilon(1 \mathrm{~S})$ is shown in FIG.3-5 and is estimated in model-I for $\left\langle k_{\perp}^{2}\right\rangle=1 \mathrm{GeV}^{2}$ and $r=\frac{1}{3}$. Rapidity distribution is obtained by integrating $p_{T} \in[0,0.5 \mathrm{GeV}]$. The small window of $p_{T}$ bin $\left(0<p_{T}<0.5\right)$ is chosen to illustrate the effect of linearly polarized gluons in unpolarized $p p$ collision. The rapidity spectrum of quarkonium obtained in COM is compared with that of CEM [20] which is shown in the same figures, for model-I. Comparatively, the production rates of $J / \psi$ and $\Upsilon(1 S)$ are slightly higher in COM than CEM. The rapidity distribution of quarkonium in model-II also follows the pattern obtained through model-I, however with less magnitude. The $p_{T}$ and $y$ distribution of quarkonium increase with increasing $k_{\max }\left(=\sqrt{\left\langle k_{\perp a}^{2}\right\rangle}\right)$ in model-II. The rapidity distribution is enhanced by inclusion of linearly polarized gluons in quarkonium production. The enhancement in the rapidity distribution is more at LHCb compared to RHIC and AFTER experiments.

The quarkonium production through COM ("Set-I" LDMEs) and CEM within TMD evolution approach using "AR" $R_{N P}$ factor are compared in FIG.6-11. $p_{T}$ distribution of quarkonium in TMD evolution approach is shown in FIG.6-8 at LHCb, RHIC and AFTER energies using Eqs.(30)-(32). The effect of linearly polarized gluons increases with center of mass energy of the process. The $p_{T}$ distribution of $J / \psi$ is greatly affected by linearly polarized gluons at $\mathrm{LHCb}$ energy compared to RHIC and AFTER energies. Nevertheless, the effect is sizable at low $p_{T}$. The effect of linearly polarized gluons is less in $\Upsilon(1 \mathrm{~S})$ production due to $\Upsilon(1 \mathrm{~S})$ mass. The rapidity spectrum of quarkonium is shown in FIG.9-11 using TMD evolution approach. Transverse momentum is integrated in the range of $0<p_{T}<4 \mathrm{GeV}$ for $y$ distribution. In TMD evolution, the production rates of $J / \psi$ and $\Upsilon(1 \mathrm{~S})$ are more in COM. However, the effect of $h_{1}^{\perp g}$ in $J / \psi$ production is significantly high in COM compared with CEM.

The effect of $h_{1}^{\perp g}$ in TMD evolution is not as much as DGLAP evolution approach. The comparison between DGLAP and TMD evolution in COM is shown in FIG.12 for $p_{T}$ spectrum of quarkonium. The bands in the figures are obtained by varying the scale (mass of quarkonium) 
from $Q=3.096 \mathrm{GeV}$ to $3.596 \mathrm{GeV}$ and $9.398 \mathrm{GeV}$ to $9.898 \mathrm{GeV}$ for $J / \psi$ and $\Upsilon(1 \mathrm{~S})$ respectively. The gluon momentum fraction, $x_{g}$, is proportional to the mass $(\mathrm{M})$ of quarkonium. Hence, the value of $x_{g}$ is large for massive quarkonium and the gluon PDF decreases very rapidly for large values of $x_{g}$. Therefore, there is not much effect on the $p_{T}$ spectrum of $\Upsilon(1 \mathrm{~S})$ due to the variation in the scale. Moreover, the effect is less in TMD evolution compared to DGLAP evolution. The reason is that in DGLAP evolution the collinear PDFs are probed at the scale $Q$ whereas PDFs are measured at the initial scale $c / b_{*}$ in TMD evolution approach. In FIG.13-18, $p_{T}$ spectrum of quarkonium obtained in TMD evolution in CEM and COM is compared with RHIC data at $\sqrt{s}=200 \mathrm{GeV}[58]$ and LHCb data at $\sqrt{s}=7 \mathrm{TeV}[59,60]$. For obtaining $p_{T}$ spectrum of quarkonium "Set-I" and "Set-II" LDMEs are considered for color octet states and two nonperturbative Sudakov factors "AR" and "BLNY" are used in TMD evolution which is shown in FIG.13-18. The theoretical prediction of $p_{T}$ spectrum of $J / \psi$ in CEM and COM using "Set-II" LDMEs is in considerable agreement with LHCb data up to low $p_{T}$ which is shown in FIG.13 and 14, whereas it is slightly underestimated for RHIC energy as shown in FIG.15 and 16. The $p_{T}$ spectrum of $\Upsilon(1 \mathrm{~S})$ is compared with the LHC data [60] and is shown in FIG.17 and 18. The obtained $\Upsilon(1 \mathrm{~S})$ production rate in CEM is in good agreement with LHC data up to 8 $\mathrm{GeV}$. However, the low $p_{T}$ region is slightly overestimated in COM using "Set-I" LDMEs for both $J / \psi$ and $\Upsilon(1 \mathrm{~S})$ production in particular for LHCb experiment. The $p_{T}$ spectrum of $\Upsilon(1 \mathrm{~S})$ is slightly underestimated in COM using "Set-II" LDMEs. The obtained $p_{T}$ spectrum of $J / \psi$ at RHIC energy using "BLNY" $R_{N P}$ is falling somewhat faster than "AR" nonperturbative parametrization. The effect of linearly polarized gluons is very less for "BLNY" compared to "AR" $R_{N P}$. In FIG.15-18, $\mathrm{B}_{e e}(0.0594)$ and $\mathrm{B}_{\mu \mu}(0.0248)$ are the branching ratios of $J / \psi \rightarrow e^{+} e^{-}$ and $\Upsilon(1 \mathrm{~S}) \rightarrow \mu^{+} \mu^{-}$channels respectively. $J / \psi$ can also be produced in addition to the direct production in $p p$ collision, for instance, decay from higher mass excited states $\left(\psi(2 \mathrm{~S})\right.$ and $\left.\chi_{c}\right)$ and decay of B-meson. The decay of $\Upsilon(2 \mathrm{~S}), \Upsilon(3 \mathrm{~S})$ and $\chi_{b}$ contribute to the $\Upsilon(1 \mathrm{~S})$ production. However, in this paper we have considered only the direct production and the inclusion of these feed down contribution to the quarkonium production is beyond the scope of this paper. Of course, leading order calculation for quarkonium production is insufficient to explain high $p_{T}$ data. It would be interesting to investigate the high $p_{T}$ spectrum of quarkonium at LO plus NLO calculation in TMD formalism. Inclusion of the so-called Y-term [61] is also expected to 
improve the behavior at high $p_{T}$.
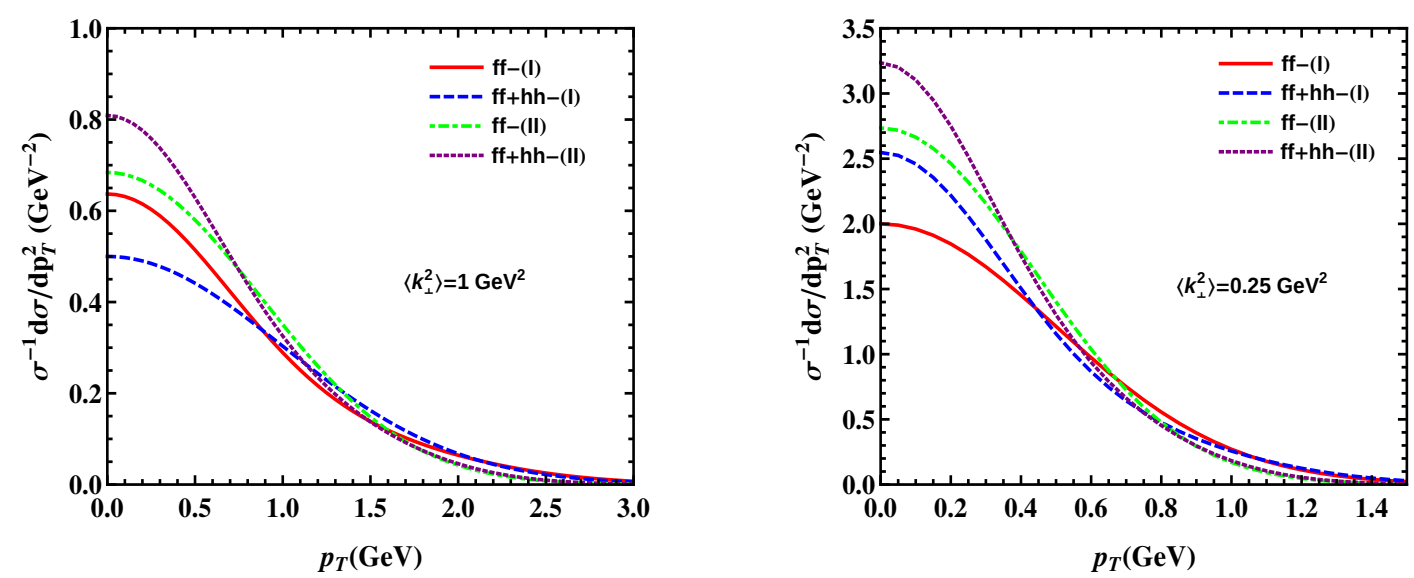

FIG. 1. (color online) Differential cross section (normalized) of $J / \psi$ and $\Upsilon(1 \mathrm{~S})$ production in $p p \rightarrow$ $J / \psi(\Upsilon(1 \mathrm{~S}))+X$ at $\operatorname{LHCb}(\sqrt{s}=7 \mathrm{TeV}), \operatorname{RHIC}(\sqrt{s}=500 \mathrm{GeV})$ and $\operatorname{AFTER}(\sqrt{s}=115 \mathrm{GeV})$ energies using "Set-I" LDMEs in DGLAP evolution approach for $r=\frac{2}{3}$. The solid (ff-(I)) and dot dashed (ff(II)) lines are obtained by considering unpolarized gluons in Model-I and Model-II respectively. The dashed (ff+hh-(I)) and tiny dashed (ff+hh-(II)) lines are obtained by taking into account unpolarized gluons plus linearly polarized gluons in Model-I and Model-II respectively. See the text for ranges of rapidity integration.
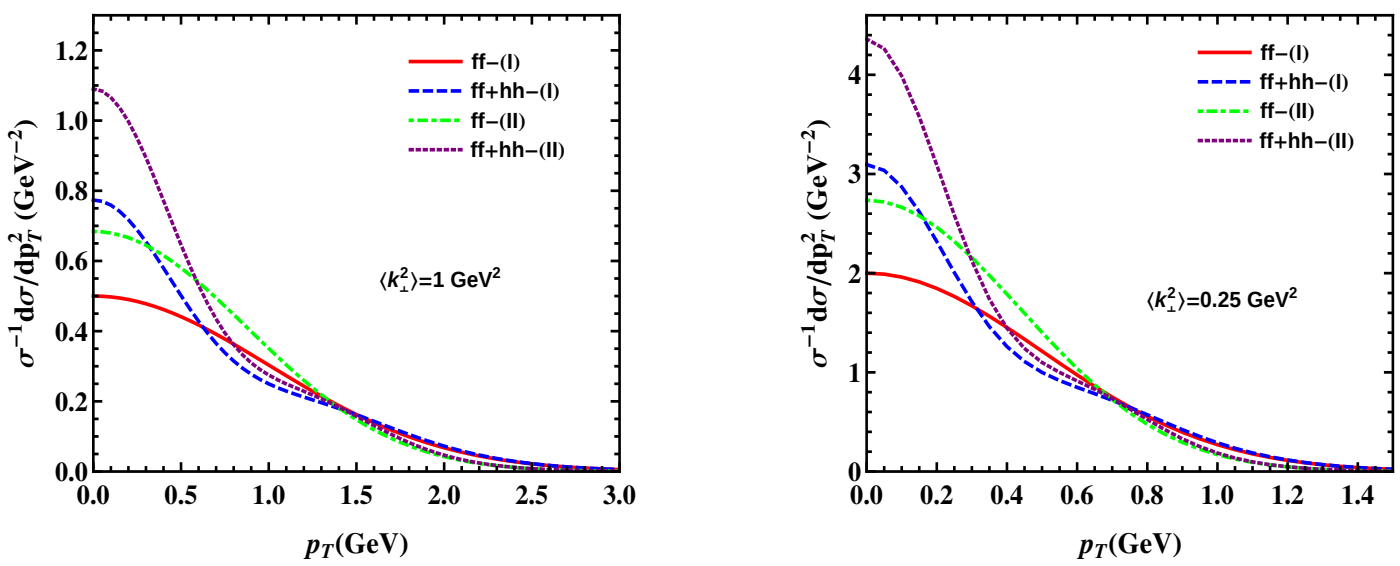

FIG. 2. (color online) Same as in Fig. 2 but for $r=\frac{1}{3}$. 


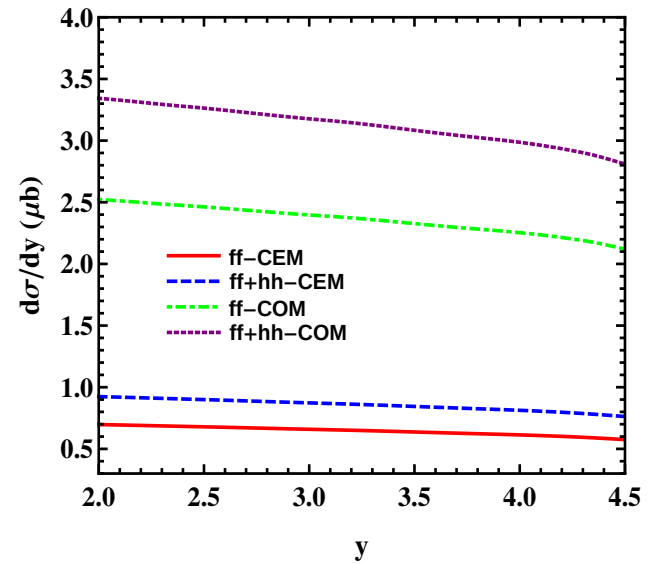

(a)

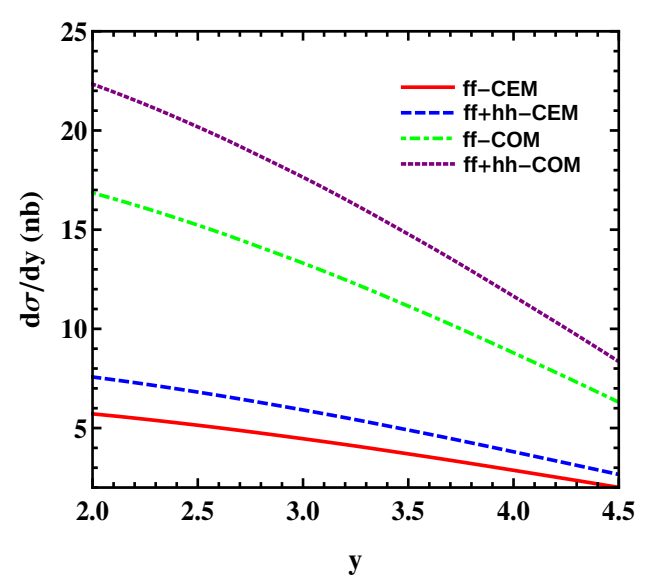

(b)

FIG. 3. (color online). Rapidity ( $y$ ) distribution of (a) $J / \psi$ (left panel) and (b) $\Upsilon(1 \mathrm{~S})$ (right panel) in $p p \rightarrow J / \psi(\Upsilon(1 \mathrm{~S}))+X$ at $\mathrm{LHCb}(\sqrt{s}=7 \mathrm{TeV})$ energy and $p_{T}$ integration range is from 0 to $0.5 \mathrm{GeV}$ using DGLAP evolution approach in Model-I for $\left\langle k_{\perp}^{2}\right\rangle=1 \mathrm{GeV}^{2}$ and $r=\frac{1}{3}$ in both CEM and COM. "Set-I" LDMEs are used in COM. The solid (ff-CEM) and dot dashed (ff-COM) lines are obtained by considering unpolarized gluons in CEM and COM respectively. The dashed (ff+hh-CEM) and tiny dashed (ff+hh-COM) lines are obtained by taking into account unpolarized gluons plus linearly polarized gluons in CEM and COM respectively.

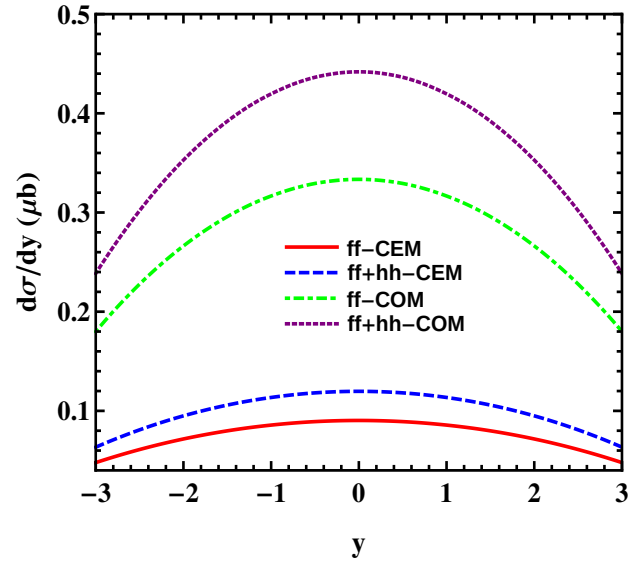

(a)

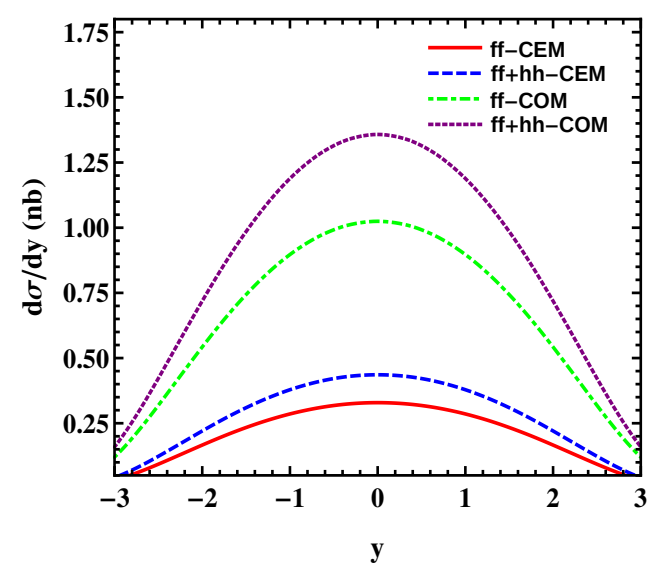

(b)

FIG. 4. (color online). Rapidity (y) distribution of (a) $J / \psi$ (left panel) and (b) $\Upsilon(1 \mathrm{~S}$ ) (right panel) in $p p \rightarrow J / \psi(\Upsilon(1 \mathrm{~S}))+X$ at RHIC $(\sqrt{s}=500 \mathrm{GeV})$ energy and $p_{T}$ integration range is from 0 to $0.5 \mathrm{GeV}$ using DGLAP evolution approach in Model-I for $\left\langle k_{\perp}^{2}\right\rangle=1 \mathrm{GeV}^{2}$ and $r=\frac{1}{3}$ in both CEM and COM. "Set-I" LDMEs are used in COM. The convention in the figure for line styles is same as Fig. 3. 


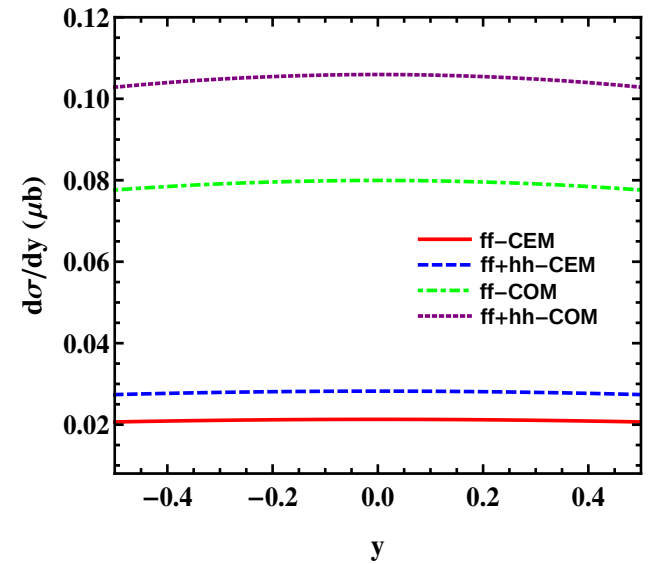

(a)

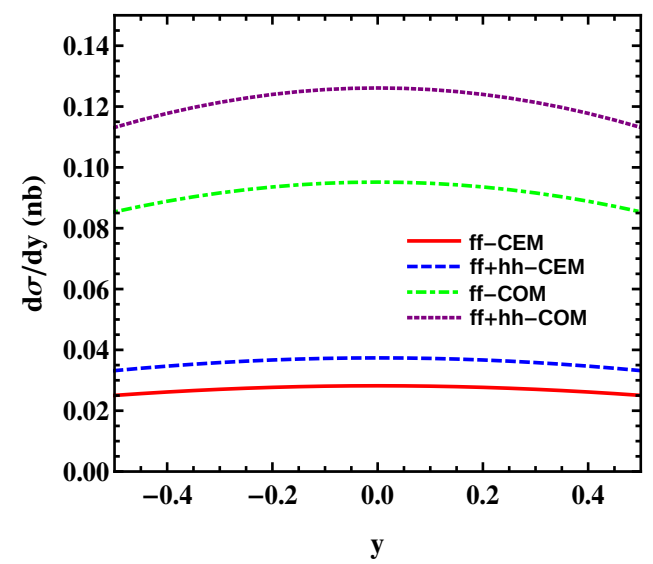

(b)

FIG. 5. (color online). Rapidity (y) distribution of (a) $J / \psi$ (left panel) and (b) $\Upsilon(1 \mathrm{~S})$ (right panel) in $p p \rightarrow J / \psi(\Upsilon(1 \mathrm{~S}))+X$ at AFTER $(\sqrt{s}=115 \mathrm{GeV})$ energy and $p_{T}$ integration range is from 0 to $0.5 \mathrm{GeV}$ using DGLAP evolution approach in Model-I for $\left\langle k_{\perp}^{2}\right\rangle=1 \mathrm{GeV}^{2}$ and $r=\frac{1}{3}$ in both CEM and COM. "Set-I" LDMEs are used in COM. The convention in the figure for line styles is same as Fig. 3.

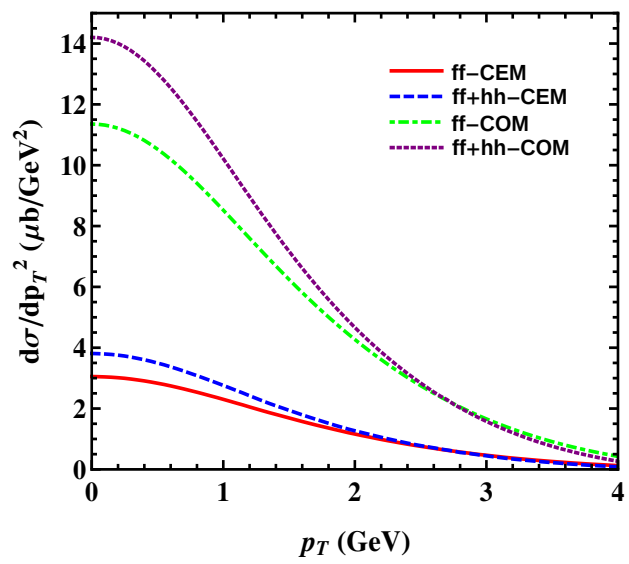

(a)

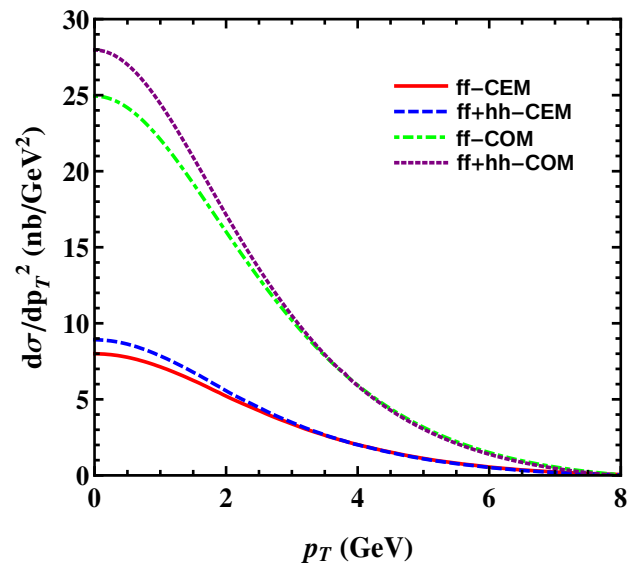

(b)

FIG. 6. (color online). Differential cross section of (a) $J / \psi$ (left panel) and (b) $\Upsilon(1 \mathrm{~S})$ (right panel) as function of $p_{T}$ in $p p \rightarrow J / \psi(\Upsilon(1 \mathrm{~S}))+X$ at $\mathrm{LHCb}(\sqrt{s}=7 \mathrm{TeV})$ energy using TMD evolution approach in CEM and COM. "Set-I" LDMEs and "AR" $R_{N P}$ are used in COM. The integration range of y is $2.0<y<4.5$. The convention in the figure for line styles is same as Fig. 3 . 


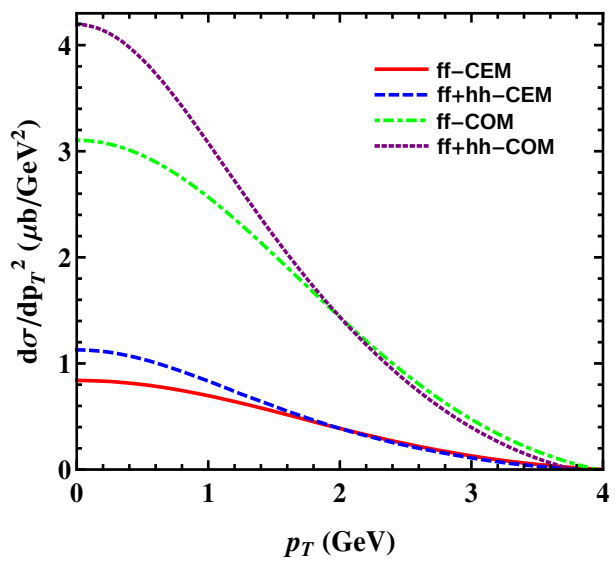

(a)

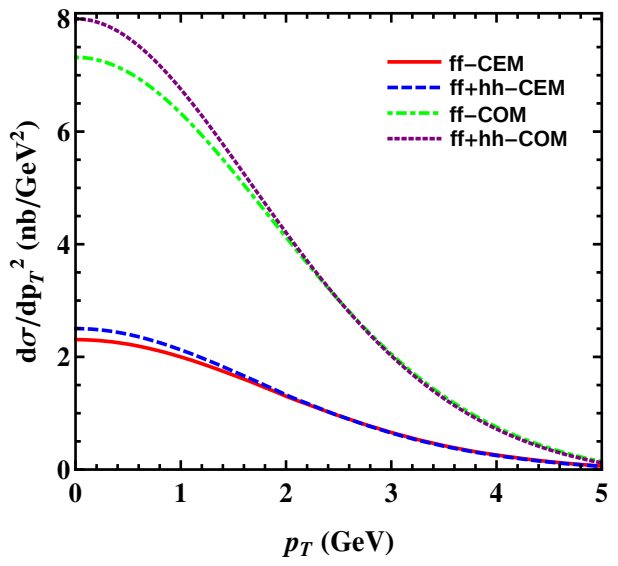

(b)

FIG. 7. (color online). Differential cross section of (a) $J / \psi$ (left panel) and (b) $\Upsilon(1 \mathrm{~S})$ (right panel) as function of $p_{T}$ in $p p \rightarrow J / \psi(\Upsilon(1 \mathrm{~S}))+X$ at RHIC $(\sqrt{s}=500 \mathrm{GeV})$ energy using TMD evolution approach in CEM and COM. "Set-I" LDMEs and "AR" $R_{N P}$ are used in COM. The integration range of $\mathrm{y}$ is $-3.0<y<3.0$. The convention in the figure for line styles is same as Fig. 3.

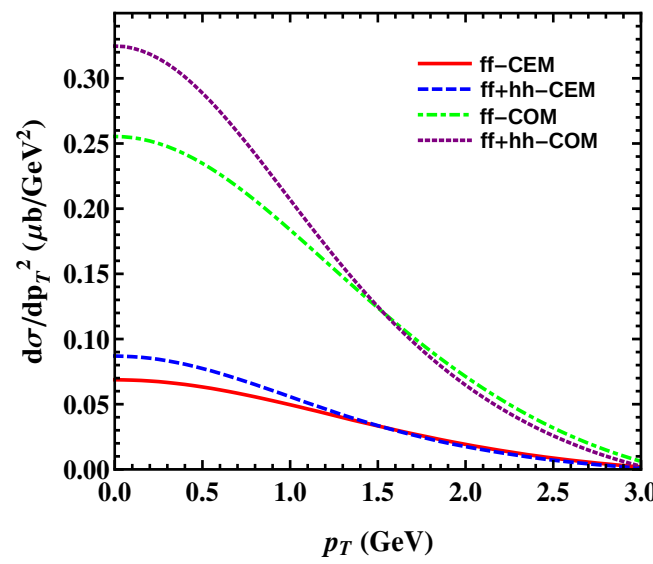

(a)

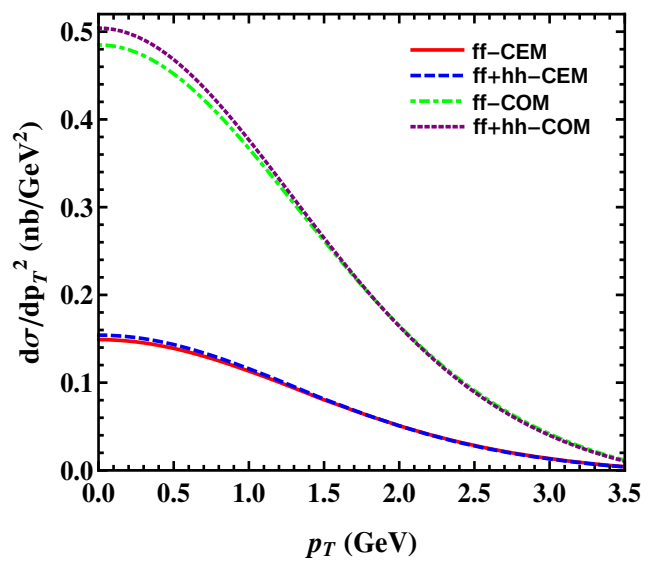

(b)

FIG. 8. (color online). Differential cross section of (a) $J / \psi$ (left panel) and (b) $\Upsilon(1 \mathrm{~S}$ ) (right panel) as function of $p_{T}$ in $p p \rightarrow J / \psi(\Upsilon(1 \mathrm{~S}))+X$ at AFTER $(\sqrt{s}=115 \mathrm{GeV})$ energy using TMD evolution approach in CEM and COM. "Set-I" LDMEs and "AR" $R_{N P}$ are used in COM. The integration range of $\mathrm{y}$ is $-0.5<y<0.5$. The convention in the figure for line styles is same as Fig. 3 . 


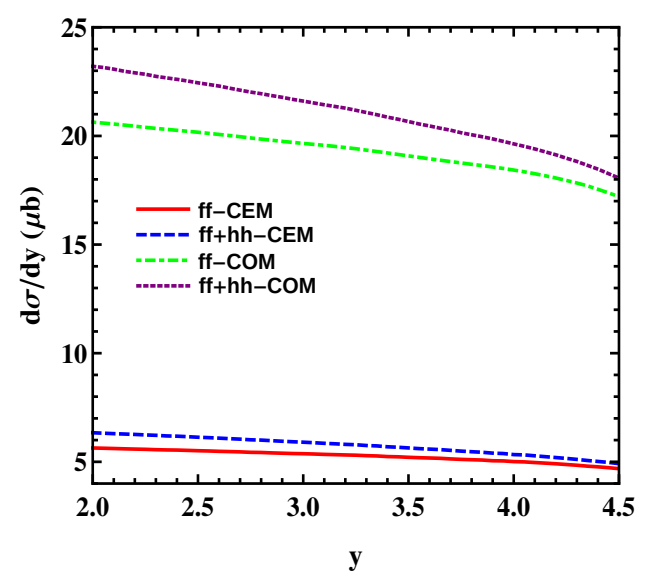

(a)

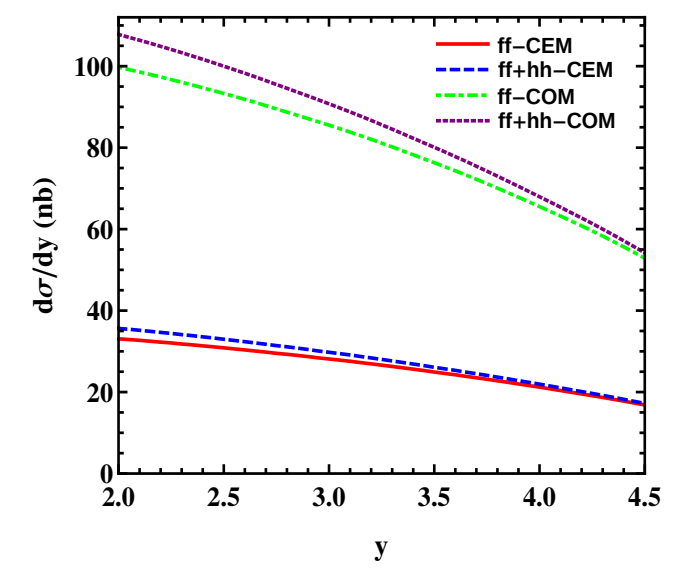

(b)

FIG. 9. (color online). Differential cross section of (a) $J / \psi$ (left panel) and (b) $\Upsilon(1 \mathrm{~S}$ ) (right panel) as function of y in $p p \rightarrow J / \psi(\Upsilon(1 \mathrm{~S}))+X$ at $\operatorname{LHCb}(\sqrt{s}=7 \mathrm{TeV})$ energy using TMD evolution approach in CEM and COM. "Set-I" LDMEs and "AR" $R_{N P}$ are used in COM. The integration range of $p_{T}$ is $0<p_{T}<4.0 \mathrm{GeV}$. The convention in the figure for line styles is same as Fig. 3.

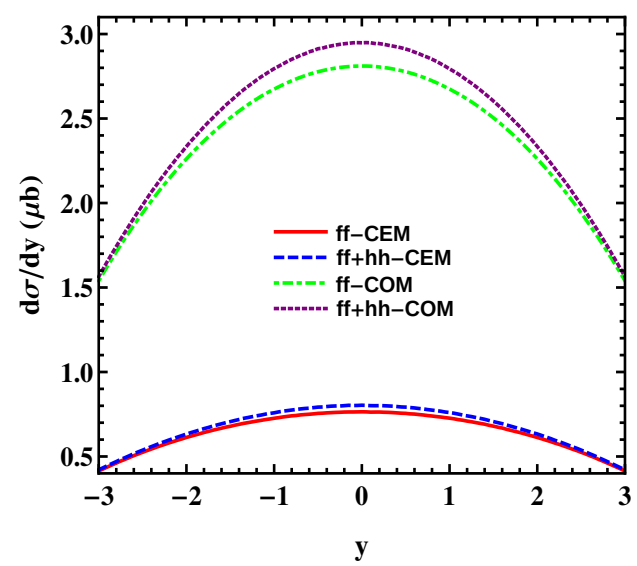

(a)

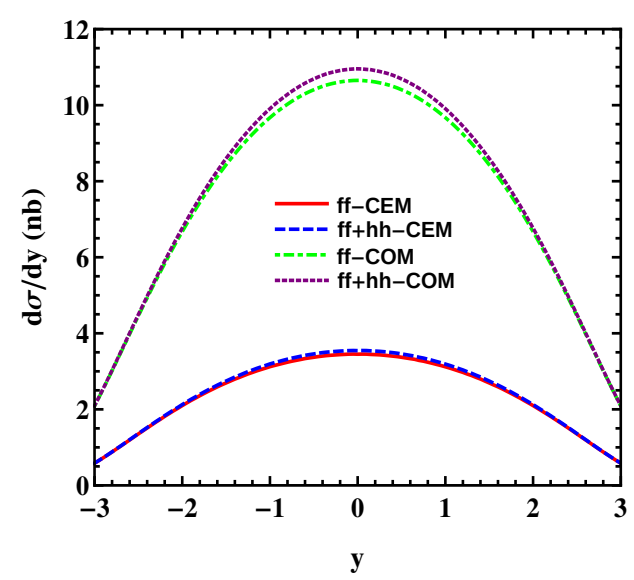

(b)

FIG. 10. (color online). Differential cross section of (a) $J / \psi$ (left panel) and (b) $\Upsilon(1 \mathrm{~S}$ ) (right panel) as function of $\mathrm{y}$ in $p p \rightarrow J / \psi(\Upsilon(1 \mathrm{~S}))+X$ at RHIC $(\sqrt{s}=500 \mathrm{GeV})$ energy using TMD evolution approach in CEM and COM. "Set-I" LDMEs and "AR" $R_{N P}$ are used in COM. The integration range of $p_{T}$ is $0<p_{T}<4.0 \mathrm{GeV}$. The convention in the figure for line styles is same as Fig. 3. 


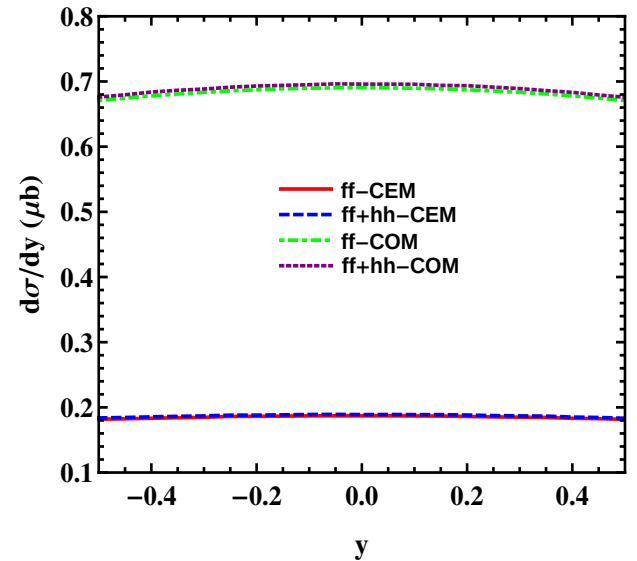

(a)

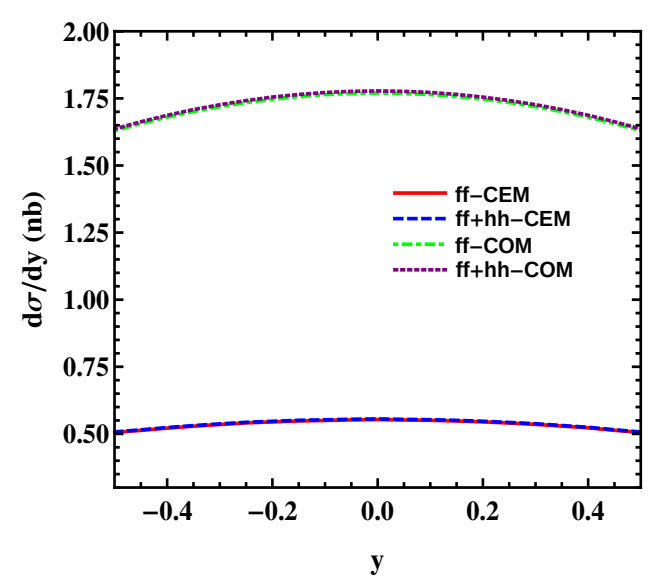

(b)

FIG. 11. (color online). Differential cross section of (a) $J / \psi$ (left panel) and (b) $\Upsilon(1 \mathrm{~S}$ ) (right panel) as function of y in $p p \rightarrow J / \psi(\Upsilon(1 \mathrm{~S}))+X$ at $\operatorname{AFTER}(\sqrt{s}=115 \mathrm{GeV})$ energy using TMD evolution approach in CEM and COM. "Set-I" LDMEs and "AR" $R_{N P}$ are used in COM. The integration range of $p_{T}$ is $0<p_{T}<4.0 \mathrm{GeV}$. The convention in the figure for line styles is same as FIG.3.

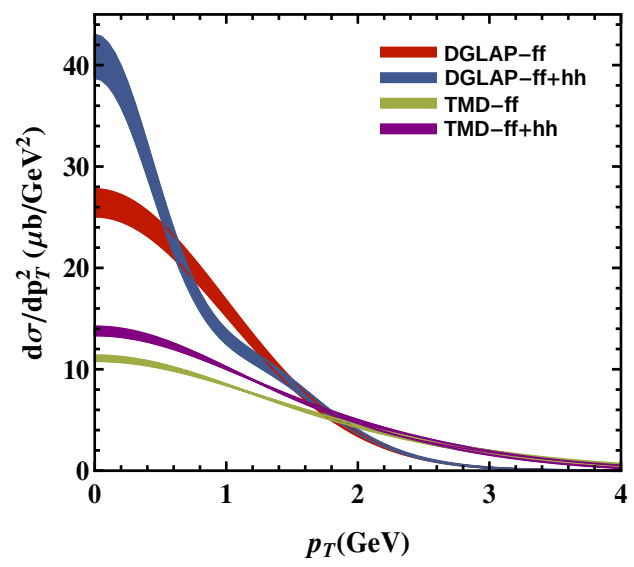

(a)

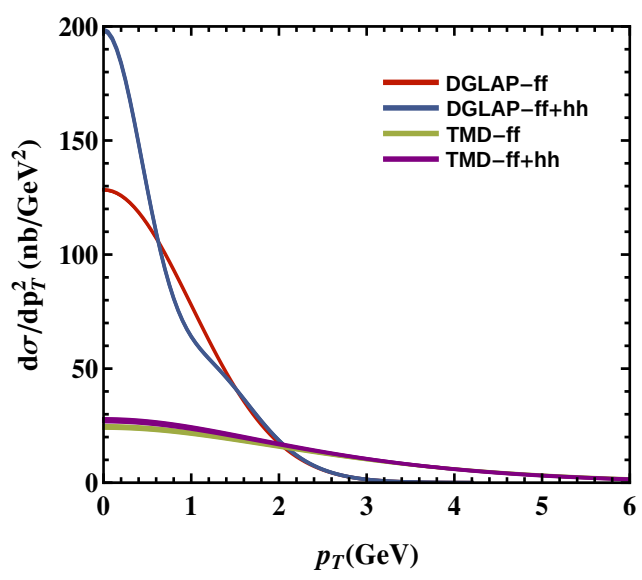

(b)

FIG. 12. (color online). Differential cross section of (a) $J / \psi$ (left panel) and (b) $\Upsilon(1 \mathrm{~S})$ (right panel) in $p p \rightarrow J / \psi(\Upsilon(1 \mathrm{~S}))+X$ at LHCb $(\sqrt{s}=7 \mathrm{TeV})$ in COM using "Set-I" LDMEs. For TMD evolution "AR" $R_{N P}$ is used. See text for the variation of the scale that is shown in bands. We have chosen $r=\frac{1}{3}$ and $\left\langle k_{\perp}^{2}\right\rangle=1 \mathrm{GeV}^{2}$ in Model-I for DGLAP evolution. 


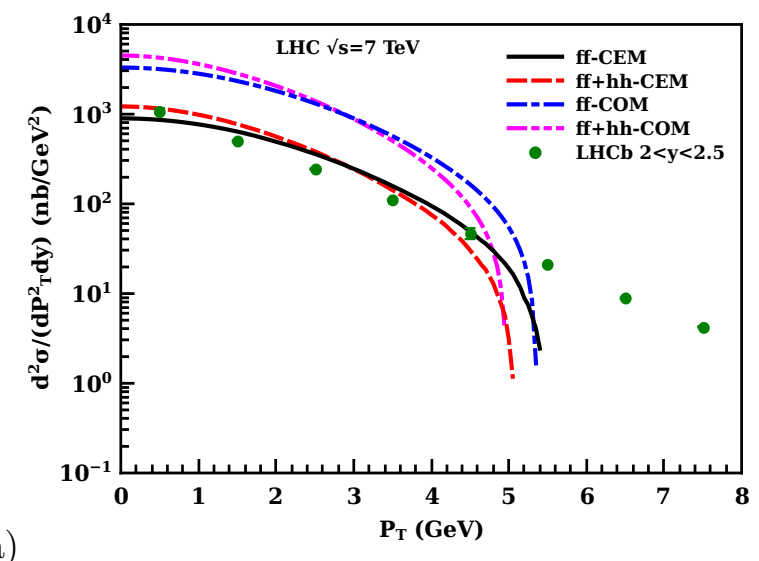

(a)

FIG. 13. (color online). Differential cross section of $J / \psi$ at $\mathrm{LHCb}(\sqrt{s}=7 \mathrm{TeV})$ as function of $p_{T}$ in $p p \rightarrow J / \psi+X$ using (a) "Set-I" (left) and (b) "Set-II" (right) LDMEs in COM within TMD evolution approach for "AR" $R_{N P}$ factor. Data is taken from [59]. The convention in the figure for line styles is same as Fig. 3. The rapidity in the range $2.0<y<2.5$ is chosen.

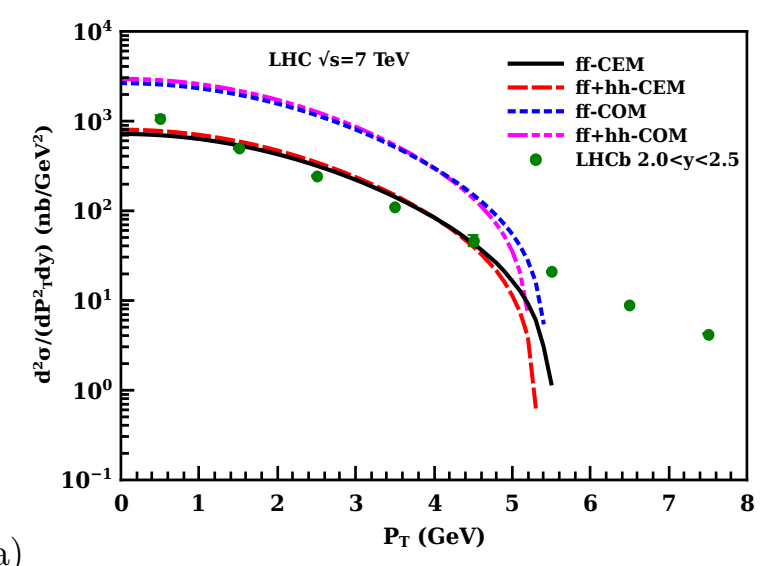

(a)

(b)

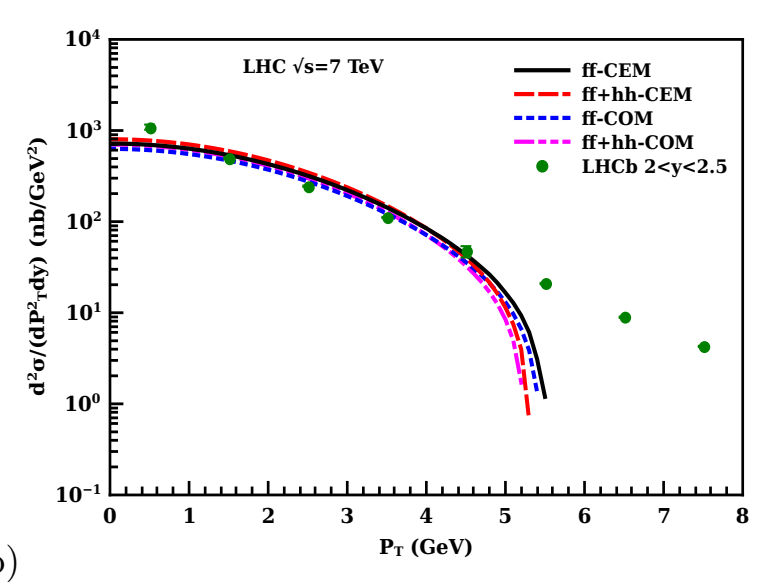

FIG. 14. (color online). Differential cross section of $J / \psi$ at LHCb $(\sqrt{s}=7 \mathrm{TeV})$ as function of $p_{T}$ in $p p \rightarrow J / \psi+X$ using (a) "Set-I" (left) and (b) "Set-II" (right) LDMEs in COM within TMD evolution approach for "BLNY" $R_{N P}$ factor. Data is taken from [59]. The convention in the figure for line styles is same as Fig. 3. The rapidity in the range $2.0<y<2.5$ is chosen. 


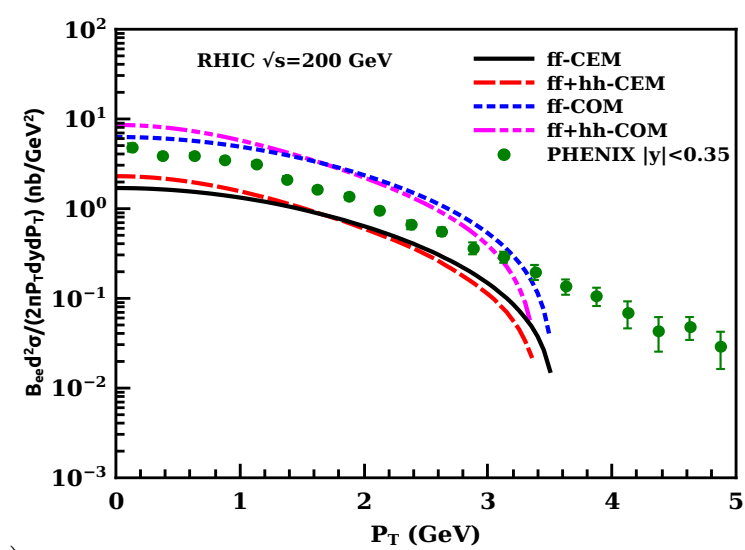

(a)

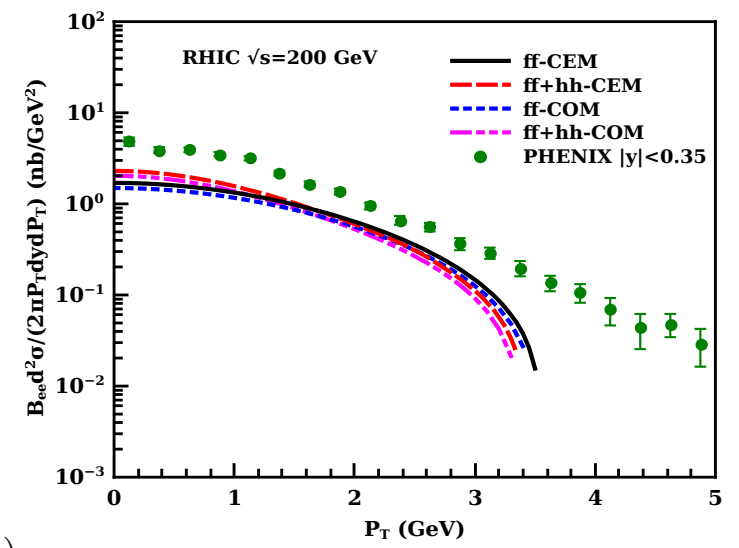

(b)

FIG. 15. (color online). Differential cross section of $J / \psi$ at RHIC $(\sqrt{s}=200 \mathrm{GeV})$ as function of $p_{T}$ in $p p \rightarrow J / \psi+X$ using (a) "Set-I" (left) and (b) "Set-II" (right) LDMEs in COM within TMD evolution approach for "AR" $R_{N P}$ factor. Data is taken from [58]. The convention in the figure for line styles is same as Fig. 3. The rapidity in the range $-0.35<y<0.35$ is chosen.

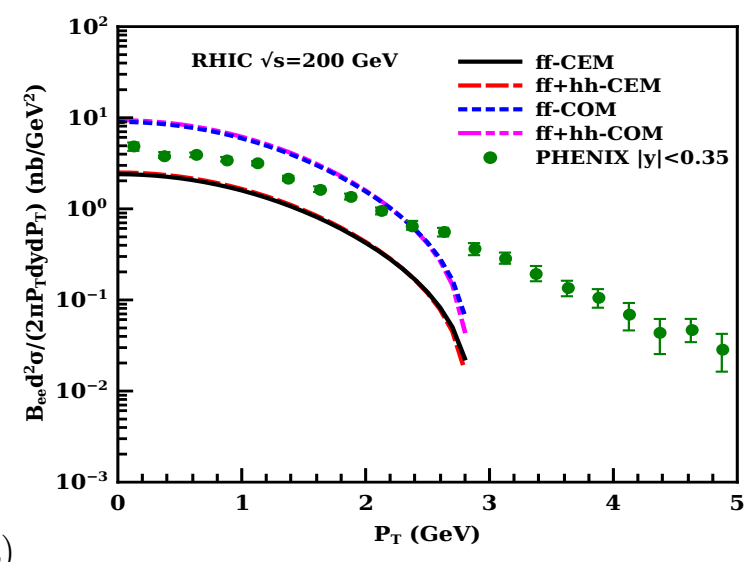

(a)

FIG. 16. (color online). Differential cross section of $J / \psi$ at RHIC $(\sqrt{s}=200 \mathrm{GeV})$ as function of $p_{T}$ in $p p \rightarrow J / \psi+X$ using (a) "Set-I" (left) and (b) "Set-II" (right) LDMEs in COM within TMD evolution approach for "BLNY" $R_{N P}$ factor. Data is taken from [58]. The convention in the figure for line styles is same as Fig. 3. The rapidity in the range $-0.35<y<0.35$ is chosen. 

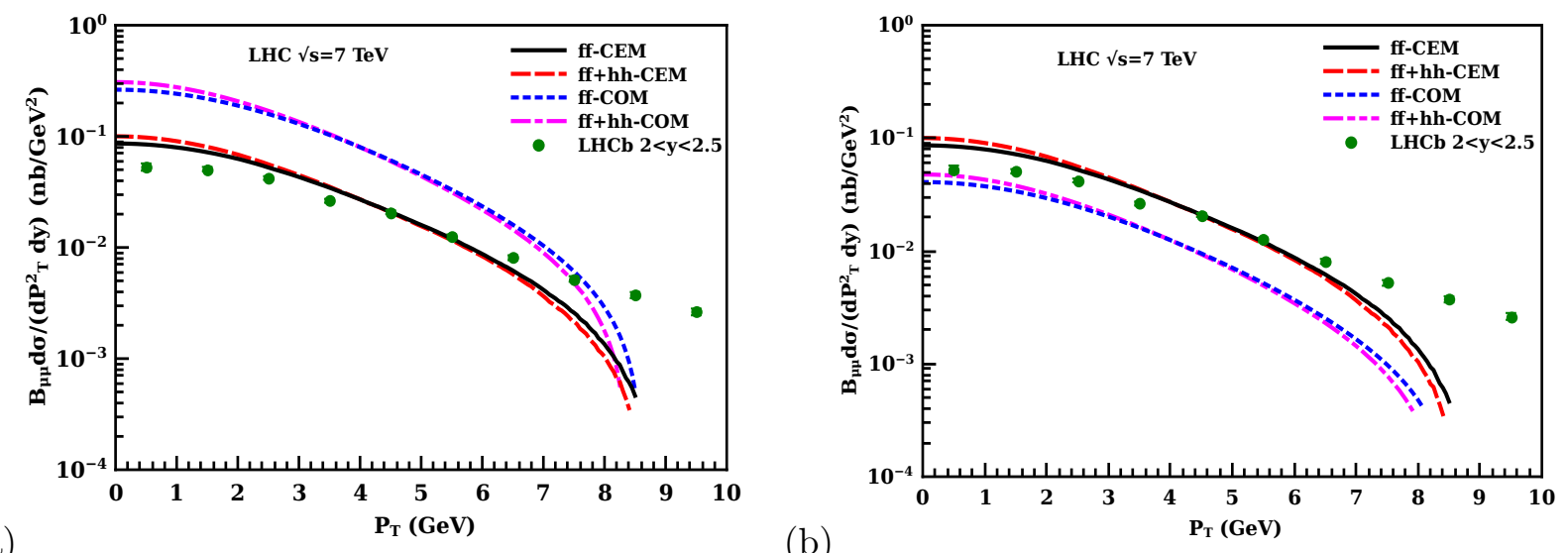

(a)

FIG. 17. (color online). Differential cross section of $\Upsilon(1 \mathrm{~S})$ ) at LHCb $(\sqrt{s}=7 \mathrm{TeV})$ as function of $p_{T}$ in $p p \rightarrow \Upsilon(1 \mathrm{~S}))+X$ using (a) "Set-I" (left) and (b) "Set-II" (right) LDMEs in COM within TMD evolution approach for "AR" $R_{N P}$ factor. Data is taken from [60]. The convention in the figure for line styles is same as Fig. 3. The rapidity in the range $2<y<2.5$ is chosen.
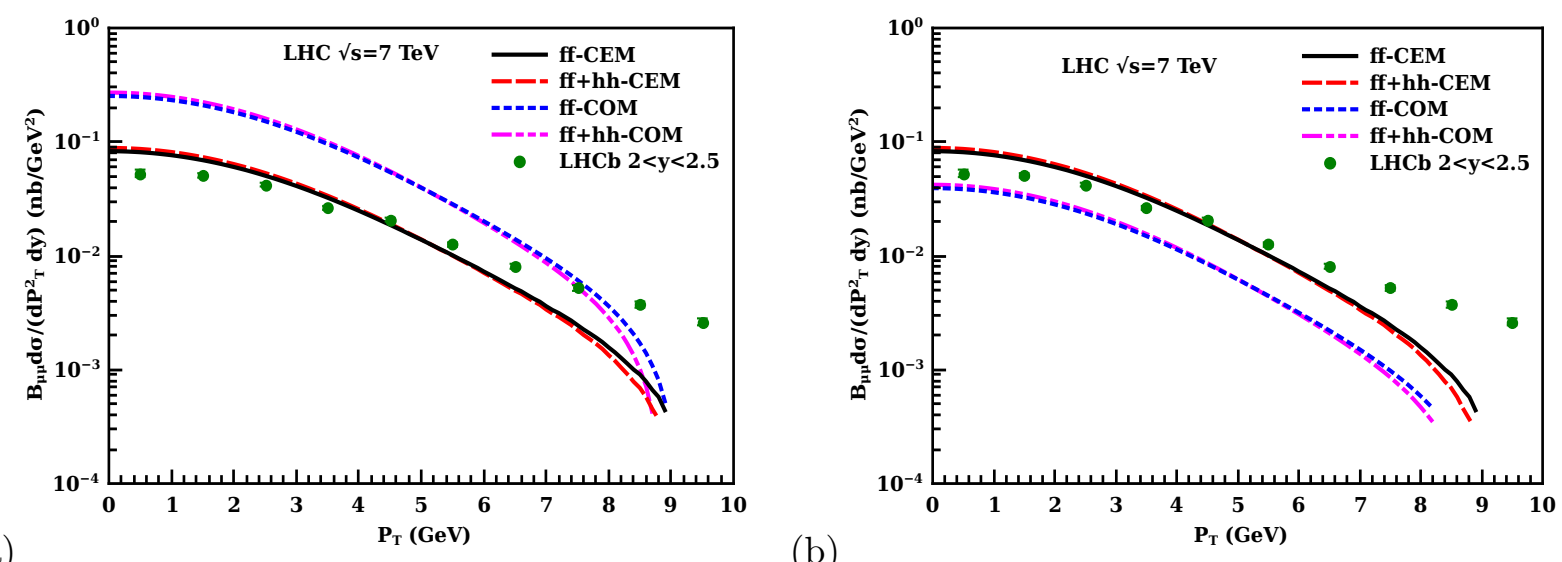

(b)

FIG. 18. (color online). Differential cross section of $\Upsilon(1 \mathrm{~S}))$ at LHCb $(\sqrt{s}=7 \mathrm{TeV})$ as function of $p_{T}$ in $p p \rightarrow \Upsilon(1 \mathrm{~S}))+X$ using (a) "Set-I" (left) and (b) "Set-II" (right) LDMEs in COM within TMD evolution approach for "BLNY" $R_{N P}$ factor. Data is taken from [60]. The convention in the figure for line styles is same as Fig. 3. The rapidity in the range $2<y<2.5$ is chosen. 


\section{CONCLUSION}

In summary, we studied the transverse momentum and rapidity distribution of $J / \psi$ and $\Upsilon(1 \mathrm{~S})$ in unpolarized proton-proton collision within non-relativistic QCD based color octet model using TMD factorization formalism. The LO color octet states ${ }^{1} S_{0},{ }^{3} P_{0}$ and ${ }^{3} P_{2}$ of the initial heavy quark pair in gluon-gluon fusion channel have been considered for quarkonium production. The quarkonium production rates are estimated at LHCb, RHIC and AFTER center of mass energies. Significant modulations in transverse momentum spectrum of $J / \psi$ are shown, in the low $p_{T}$ region, when contribution from linearly polarized gluons inside an unpolarized proton is included. The rapidity distribution is enhanced with the inclusion of $h_{1}^{\perp g}$. The obtained production rates of quarkonium in COM are compared with CEM. The effect of $h_{1}^{\perp g}$ in $\Upsilon(1 S)$ production is not as dominant as in $J / \psi$ production. Thus quarkonium production is a very useful process to extract both unpolarized and linearly polarized gluon density functions. However, one has to understand the quarkonium production mechanism for a reliable extraction of the TMDs. The theoretical predictions of $J / \psi$ and $\Upsilon$ in NRQCD based color octet model are in good agreement with the LHC and RHIC data up to low $p_{T}$.

[1] J. Collins, Foundations of perturbative QCD (Cambridge University Press, 2013), URL http: //www.cambridge.org/de/knowledge/isbn/item5756723.

[2] K. Kanazawa, Y. Koike, A. Metz, and D. Pitonyak, Phys. Rev. D91, 014013 (2015), 1410.3448.

[3] L. Gamberg, Z.-B. Kang, A. Metz, D. Pitonyak, and A. Prokudin, Phys. Rev. D90, 074012 (2014), 1407.5078.

[4] R. Angeles-Martinez et al., Acta Phys. Polon. B46, 2501 (2015), 1507.05267.

[5] A. Kulesza, G. F. Sterman, and W. Vogelsang, Phys. Rev. D66, 014011 (2002), hep-ph/0202251.

[6] V. Barone, F. Bradamante, and A. Martin, Prog. Part. Nucl. Phys. 65, 267 (2010), 1011.0909.

[7] U. D’Alesio and F. Murgia, Prog. Part. Nucl. Phys. 61, 394 (2008), 0712.4328.

[8] S. Melis, EPJ Web Conf. 85, 01001 (2015), 1412.1719.

[9] W. J. den Dunnen, D. Boer, C. Pisano, M. Schlegel, and W. Vogelsang, in Proceedings, 20th International Workshop on Deep-Inelastic Scattering and Related Subjects (DIS 2012): Bonn, 
Germany, March 26-30, 2012 (2012), pp. 997-1000, [,997(2012)], 1205.6931, URL https: //inspirehep.net/record/1116656/files/arXiv:1205.6931.pdf.

[10] P. J. Mulders and J. Rodrigues, Phys. Rev. D63, 094021 (2001), hep-ph/0009343.

[11] D. Boer, S. J. Brodsky, P. J. Mulders, and C. Pisano, Phys. Rev. Lett. 106, 132001 (2011), 1011.4225 .

[12] C. Pisano, D. Boer, S. J. Brodsky, M. G. A. Buffing, and P. J. Mulders, JHEP 10, 024 (2013), 1307.3417.

[13] J.-W. Qiu, M. Schlegel, and W. Vogelsang, Phys. Rev. Lett. 107, 062001 (2011), 1103.3861.

[14] W. J. den Dunnen, J. P. Lansberg, C. Pisano, and M. Schlegel, Phys. Rev. Lett. 112, 212001 (2014), 1401.7611.

[15] D. Boer, W. J. den Dunnen, C. Pisano, M. Schlegel, and W. Vogelsang, Phys. Rev. Lett. 108, 032002 (2012), 1109.1444.

[16] M. G. Echevarria, T. Kasemets, P. J. Mulders, and C. Pisano, JHEP 07, 158 (2015), 1502.05354.

[17] D. Boer, W. J. den Dunnen, C. Pisano, and M. Schlegel, Phys. Rev. Lett. 111, 032002 (2013), 1304.2654 .

[18] D. Boer and C. Pisano, Phys. Rev. D91, 074024 (2015), 1412.5556.

[19] D. Boer and C. Pisano, Phys. Rev. D86, 094007 (2012), 1208.3642.

[20] A. Mukherjee and S. Rajesh, Phys. Rev. D93, 054018 (2016), 1511.04319.

[21] Y.-Q. Ma, R. Venugopalan, and H.-F. Zhang, Phys. Rev. D92, 071901 (2015), 1503.07772.

[22] Y.-Q. Ma and R. Venugopalan, Phys. Rev. Lett. 113, 192301 (2014), 1408.4075.

[23] J. F. Amundson, O. J. P. Eboli, E. M. Gregores, and F. Halzen, Phys. Lett. B372, 127 (1996), hep-ph/9512248.

[24] J. F. Amundson, O. J. P. Eboli, E. M. Gregores, and F. Halzen, Phys. Lett. B390, 323 (1997), hep-ph/9605295.

[25] E. L. Berger and D. L. Jones, Phys. Rev. D23, 1521 (1981).

[26] R. Baier and R. Ruckl, Phys. Lett. B102, 364 (1981).

[27] R. Baier and R. Ruckl, Nucl. Phys. B201, 1 (1982).

[28] J. E. Augustin et al. (SLAC-SP-017), Phys. Rev. Lett. 33, 1406 (1974), [Adv. Exp. Phys.5,141(1976)]. 
[29] J. J. Aubert et al. (E598), Phys. Rev. Lett. 33, 1404 (1974).

[30] G. A. Schuler, Ph.D. thesis, CERN (1994), hep-ph/9403387, URL http://documents.cern.ch/ cgi-bin/setlink?base=preprint\&categ=hep-ph\&id=9403387.

[31] P. Artoisenet, J. P. Lansberg, and F. Maltoni, Phys. Lett. B653, 60 (2007), hep-ph/0703129.

[32] J. M. Campbell, F. Maltoni, and F. Tramontano, Phys. Rev. Lett. 98, 252002 (2007), hep$\mathrm{ph} / 0703113$.

[33] F. Halzen, Phys. Lett. B69, 105 (1977).

[34] F. Halzen and S. Matsuda, Phys. Rev. D17, 1344 (1978).

[35] H. Fritzsch, Phys. Lett. B67, 217 (1977).

[36] G. T. Bodwin, E. Braaten, and G. P. Lepage, Phys. Rev. D46, R1914 (1992), hep-lat/9205006.

[37] F. Cooper, M. X. Liu, and G. C. Nayak, Phys. Rev. Lett. 93, 171801 (2004), hep-ph/0402219.

[38] S. Fleming and I. Maksymyk, Phys. Rev. D54, 3608 (1996), hep-ph/9512320.

[39] G. T. Bodwin, E. Braaten, and G. P. Lepage, Phys. Rev. D51, 1125 (1995), [Erratum: Phys. Rev.D55,5853(1997)], hep-ph/9407339.

[40] D. Boer, P. J. Mulders, and C. Pisano, Phys. Lett. B660, 360 (2008), 0712.0777.

[41] M. Anselmino, U. D'Alesio, and F. Murgia, Phys. Rev. D67, 074010 (2003), hep-ph/0210371.

[42] P. L. Cho and A. K. Leibovich, Phys. Rev. D53, 6203 (1996), hep-ph/9511315.

[43] P. L. Cho and A. K. Leibovich, Phys. Rev. D53, 150 (1996), hep-ph/9505329.

[44] K.-T. Chao, Y.-Q. Ma, H.-S. Shao, K. Wang, and Y.-J. Zhang, Phys. Rev. Lett. 108, 242004 (2012), 1201.2675.

[45] R. Sharma and I. Vitev, Phys. Rev. C87, 044905 (2013), 1203.0329.

[46] B. Gong, L.-P. Wan, J.-X. Wang, and H.-F. Zhang, Phys. Rev. Lett. 110, 042002 (2013), 1205.6682 .

[47] N. Brambilla et al., Eur. Phys. J. C74, 2981 (2014), 1404.3723.

[48] B. Gong, L.-P. Wan, J.-X. Wang, and H.-F. Zhang, Phys. Rev. Lett. 112, 032001 (2014), 1305.0748.

[49] M. Anselmino, M. Boglione, U. D’Alesio, A. Kotzinian, S. Melis, F. Murgia, A. Prokudin, and C. Turk, Eur. Phys. J. A39, 89 (2009), 0805.2677.

[50] S. M. Aybat and T. C. Rogers, Phys. Rev. D83, 114042 (2011), 1101.5057. 
[51] S. M. Aybat, A. Prokudin, and T. C. Rogers, Phys. Rev. Lett. 108, 242003 (2012), 1112.4423.

[52] D. Boer and W. J. den Dunnen, Nucl. Phys. B886, 421 (2014), 1404.6753.

[53] A. Idilbi, X.-d. Ji, and F. Yuan, Nucl. Phys. B753, 42 (2006), hep-ph/0605068.

[54] P. Sun, C. P. Yuan, and F. Yuan, Phys. Rev. D88, 054008 (2013), 1210.3432.

[55] A. D. Martin, W. J. Stirling, R. S. Thorne, and G. Watt, Eur. Phys. J. C63, 189 (2009), 0901.0002.

[56] A. D. Frawley, T. Ullrich, and R. Vogt, Phys. Rept. 462, 125 (2008), 0806.1013.

[57] C. P. Smith, Ph.D. thesis, University of Wisconsin (2000), URL http://www.dtic.mil/dtic/ tr/fulltext/u2/a377773.pdf.

[58] A. Adare et al. (PHENIX), Phys. Rev. D82, 012001 (2010), 0912.2082.

[59] R. Aaij et al. (LHCb), Eur. Phys. J. C71, 1645 (2011), 1103.0423.

[60] R. Aaij et al. (LHCb), Eur. Phys. J. C72, 2025 (2012), 1202.6579.

[61] J. Collins, L. Gamberg, A. Prokudin, T. C. Rogers, N. Sato, and B. Wang, Phys. Rev. D94, 034014 (2016), 1605.00671. 